\title{
Quasi una catabasi. La Divina Commedia nella danza degli ultimi 200 anni. Parte I
}

\author{
Roberto Fratini \\ Institut del Teatre \\ serafide@yahoo.it
}

\section{Riassunto}

Il saggio ripercorre due secoli di adattamenti coreografici della Divina Commedia (nell'ambito della danza classica, moderna e contemporanea), mettendoli in rapporto con la storia generale del ruolo di Dante nell'immaginario culturale d'Occidente, e sussumendone l'ipotesi di un uso generale di Dante come sintomo o catalizzatore di inquietudini epocali, paradossi formali e "vizi" poetici della danza degli ultimi duecento anni.

Parole chiave: Dante; Commedia; danza; balletto.

\begin{abstract}
The essay retraces two centuries of choreographic adaptations of the Divine Comedy (in the sphere of classical, modern and contemporary dance), putting them in relation with the general history of Dante's role in the cultural imaginary of the West, and subsuming the hypothesis of a general use of Dante as a symptom or catalyst of contemporary concerns, formal paradoxes and poetic "vices" of the dance of the last two hundred years.
\end{abstract}

Keywords: Dante; Comedy; dance; ballet. 
"La palude è la salvezza o la perdizione? Non lo so, nessuno lo sa; in lei ci si riconosce e ci si smarrisce; la fine è troppo simile alla conclusione, l'inizio è il cominciamento."

Giorgio Manganelli, La palude definitiva

\section{QuAsi UNA SELVA OSCURA}

$\mathrm{U}$ na Storia di Dante nella danza degli ultimi due secoli ${ }^{1}$ dovrebbe ragionevolmente articolarsi in due parti: una che si occupi di relazioni "dirette", cioè dei casi in cui la prassi coreografica inscena una "versione" della Commedia, difendendo con mezzi poetici (e con armi esegetiche non sempre impeccabili) il suo arrembaggio al poema; e una che si occupi di interferenze involontarie, cioè degli spettacoli, poetiche, formati e pedagogie in cui, senza invocare necessariamente il modello dantesco, si sia plasmata a sua volta l'ipotesi generale di una rappresentazione danzata dell'Oltremondo, quando non l'effigie della danza come Oltremondo in sé. All'analisi di questi Aldilà danzanti, il paradigma "esogeno" della Divina Commedia offrirebbe strumenti incomparabilmente più efficaci (e certamente più divertenti) che buona parte dell'attuale teoria "endogena" della danza. Consentirebbe di ripercorrere lo stupefacente lignaggio di "escatologie danzate" che costituiscono, per tutto dire, un canone possibile della danza contemporanea (da Café Müller di Pina Bausch, a May B di Maguy Marin, a Aliena(c)tion di William Forsythe) - laddove il catalogo delle creazioni direttamente ispirate a Dante non offre che sporadicamente contributi artistici di spicco. Ma poiché l'obiettivo di questo studio è ricostruire la storia specifica delle Commedie danzate, ad esso mi atterrò, concedendomi periodicamente di trovare riscontro ai dilemmi e paradossi ispirati da Dante alla parte di danza che se ne è occupata, nei cerchi superiori di quella danza che lo ha, almeno in apparenza, ignorato.

Come ho cercato di dimostrare altrove (Fratini 20I4), la Divina Commedia è sufficientemente ricca in suggestioni cinetiche, immagini dinamiche e metafore danzistiche da contenere in embrione un'archeologia figurale della danza d'Occidente. Non stupisce che, avendo esitato tanto a scoprirne il potenziale, la danza postmoderna ne abbia abusato così sfacciatamente (una schiacciante maggioranza degli allestimenti di cui mi occupo sono posteriori al 2005). Al divenire (in danza e non solo) una delle voghe tematiche del nuovo millennio, Dante è così transitato per approcci, stilemi e malintesi ricorrenti che sembra-

I. Questo studio si è sviluppato nell'ambito del progetto di ricerca “Traza y figura de la danza en la larga Edad Media. Corpus iconográfico, textual y etnográfico en la Peninsula Ibérica y su proyección latinoamericana (FFI2OI3-42939-P)". Per una lista estesa della mole di adattamenti moderni della materia dantesca, soprattutto per quel che concerne il teatro musicale, cfr. La Salvia 20II, e il documento online della Deutsche Dante Gesellschaft (aggiornato al 2013) "Anhang: Aktuelle Neubearbeitungen der Divina Commedia". 
no meno rappresentativi della cosmovisione dantesca, che dei problemi e malesseri della danza stessa. Non vi è quasi una versione danzata della Commedia che non risulti velatamente, ma anche fatalmente, metadiscorsiva.

Come altri filoni tematici, quello dantesco non va esente da vezzi frequenti e soluzioni brevettate, che sono il riflesso di una pervicace vulgata su Dante, e del sontuoso corredo di pregiudizi di cui si è vestito nell'ultimo secolo l'immaginario collettivo attorno al poema: l'idea, per cominciare, che l'Inferno sia per colorismo e drammaticità la Cantica più teatralmente appetibile (quella, pertanto, che gode del massimo share di allestimenti. Molti dei lavori dal titolo Commedia, Divina Commedia o Dante, saranno semplicemente "inferni", con o senza supplementi purgatoriali e celestiali); che insomma la sua natura "gestuale" e spasmodica ne faccia una materia danzabile di prima scelta; il conseguente imbarazzo di fronte al Purgatorio, cassato il più delle volte (in linea con l'inveterata vulgata liceale che lo considera "noioso"); la forte tendenza, davanti ai paradossi dinamici del Paradiso, a sintetizzarlo in cosmologie zuccherose, neo-angelismi e sferologie di ogni tipo (ruote, girandole e palloni gonfiabili saranno i petulanti habitués dei Paradisi danzati); la rilevanza smodata dell'episodio di Paolo e Francesca; l'eclissi sommaria di Virgilio, in favore dell'avvenente e coreograficamente palpabile Beatrice. Vezzi o vizi, fors'anche "ideologie", utili a sceverare con esattezza la genuinità dei pochi adattamenti rilevanti, che saranno quasi sempre traslazioni, trasposizioni o "astrazioni" della Divina Commedia, espressive - per giusto o sbagliato che sia - di un gesto esegetico: di una presa di posizione sul mondo e sulla danza.

La spensierata aneddoticità con cui il balletto ottocentesco ha parassitato la Commedia non si presenta neppure come la risposta a una "questione ermeneutica". La questione, preparata dal Romanticismo letterario, dall'apoteosi della figura di Dante nei pantheon mentali della Nazione, e dal crescente auge europeo di un'idea d'opera d'arte totale atta a fomentare innumerevoli fughe retoriche verso le epopee di ogni tipo, sorge con la modernità, quando un clima culturale di "canonizzazione della Divina Commedia" invita per la prima volta a una certa prudenza in materia di saccheggio poetico. Gli anni in cui il poeta sacro per eccellenza assurge a referente assoluto di una nuova Ecumene chiamata Cultura, sono anche, non a caso, quelli del massiccio processo di sacralizzazione dell'idea di danza: di una svolta spiritualizzante che coincide sotto ogni aspetto con la palingenesi o nascita tout court di un genere che si credette dannatamente serio nel momento stesso in cui l'Occidente moderno riconosceva a Dante il privilegio di una pertinenza incalcolabile. Sincronismo che se pure, per ragioni di configurazione poetica (resistenza profilattica alla narrazione quando non alla teatralità tout court, pretesa di eloquenza somatica; pedigree di religiosità immanente), non si lascia delibare nella condotta storica della danza proto-moderna, rivelerà la sua pertinenza nel caso della 
danza post-moderna: la convergenza tardiva tra la danza del '9oo e il più sacro dei poemi non potrà darsi insomma che nel quadro di una demistificazione generale (o crisi vocazionale) della danza stessa, e della sua affannosa ricerca di nuovi titoli di legittimazione. Nello sbando di un progetto chiamato modernità, Dante sarà per così dire la religione succedanea, il feticcio après coup di una danza che non sa più essere feticcio di se stessa.

È comprensibile, sorta com'è da uno shock anafilattico di fronte all'eclissi della modernità, che la recente "febbre dantesca" si cronicizzi in armonia con el corso della post-modernità recente, cioè come la progressione geometrica di un malessere culturale dall'insorgenza alla metastasi; o come la deriva insensibile dei programmi utopici (fino agli anni '9o la nozione di post-modernità fu oggetto, anche in danza, di un certo ottimismo) a una distopia di autorealizzazioni grottesche, banalizzazioni e inflazioni. L'agenda surrettizia delle Commedie danzate con accanimento nel crepuscolo del secolo sarà così, di volta in volta, l'istituzionalizzazione dello specifico (la danza come agente culturale privilegiato), la riesumazione di antichi massimalismi (la danza come "linguaggio totale") o il ritorno a vecchi programmi salvifici o terapeutici (la danza come ultima ecumene, panacea olistica e formato spirituale definitivo): obiettivi che aiutano a comprendere la forza del delirio culturale che a innumerevoli coreografi di tutte le latitudini, negli ultimi 50 anni, ha suggerito che Dante potesse essere un interlocutore ottimale; il santo cui votare l'incombente eclissi storica della danza, o il suo frenetico mito di durevolezza.

\section{DANTe: Quasi un Balletto}

Benché il flirt recidivo tra la danza contemporanea e Dante sia complessivamente una deliquescenza della post-modernità, non sarà inutile risalire ai casi classici e neoclassici: ${ }^{2}$ la succinta genia di allestimenti che hanno configurato negli ultimi due secoli un precedente di "ballettificazione" della materia della Commedia.

2. Per l'elaborazione di un elenco (per non dire di un canone) delle creazioni coreografiche che, negli ultimi due secoli, si sono dichiaratamente ispirate alla Divina Commedia o a parti di essa, ho potuto contare con differenti rassegne nominali o cataloghi di titoli inerenti allo stesso argomento. Sapendo bene quale limite comportasse appoggiarsi unicamente a questi repertori difficilmente aggiornati per un tema che, soprattutto negli ultimi anni, ha a vario titolo goduto di una straordinaria popolarità, ho dovuto integrare quelle segnalazioni con una ricerca minuziosa di "occorrenze" dantesche nella danza degli ultimi 50 anni attraverso Internet. Precisamente la Rete, in assenza di una letteratura scientifica articolata sull'oggetto di questa analisi, è stata anche la fonte principale degli scritti d'occasione (critiche professionali e più raramente analisi dettagliate) che, accanto ai video integrali o parziali, mi hanno fornito buona parte dell'informazione imprescindibile sul contenuto delle coreografie. Tra i repertori di altro tipo cui ho attinto l'informazione basica dell'esistenza di lavori su Dante in danza classica, moderna e contemporanea, vi sono Braida-Calè 2007, Lansing 2010 e il sito: https://research.bowdoin.edu/dante-today/category/performing-arts. 
L'empirismo spregiudicato che regge la produzione ballettistica del XIX secolo non risparmia a Dante il trattamento riservato a tutte le fonti eminenti: sfruttarlo - soprassedendo sulla coerenza filologica, sulla pregnanza teologica e sull'architettura narratologica - come un repertorio di "casi"; nel caso specifico, derogare alla cornice ultramondana della Commedia astraendone l'aneddotica più confacente al codice somatico e sentimentale del balletto, passibile di un'affabulazione sufficientemente cospicua da giustificarne l'estensione su due o tre atti. Dati i penchants del balletto romantico in Italia (dove esiste una volontà tangibile di rispondere con temi nazionali alle Silfidi, Ondine, Péris e Villi delle stagioni parigine); data altresì la propagazione di una tecnica di punte che conta, nell'albo delle ballerine italiane, su rappresentanti di indubbio prestigio, s'intende che, tra le digressioni offerte dal poema, la faccia da padrona la materia amorosa; di qui che Francesca da Rimini assurga a protagonista indiscussa della tradizione, in balletto e in melodramma, che si compiace recidivamente di trasformare la lussuriosa del V Canto in eroina gemebonda e in dolente campionessa di Adagi. La danza italiana le dedicherà, in anticipo persino sull'abuso operistico del soggetto, una estenuante sequenza di balletti. ${ }^{3}$

Se l'avvinghiamento degli amanti nella bufera infernale può ben ispirare un bell'esemplare di Grand pas de Deux, è soprattutto al matrimonio infelice di Francesca, alla sua infatuazione e morte prematura che i librettisti si rifanno con candida ostinazione. Il malinteso romantico che accorda a Paolo e Francesca la prelazione su ogni ipotesi di Commedia danzata perdurerà ben oltre il repertorio ottocentesco: Paoli e Francesche di tutte le temperature erotiche imperverseranno con invincibile anacronismo persino nel contesto più dichiaratamente astratto o esegeticamente accurato di certe versioni contemporanee. Il primato degli amanti d'Oltretomba si vedrà infranto solo dallo spostamento (talora assai goffo) della tensione amorosa sul duo Dante-Beatrice. Gli adattamenti ottocenteschi condividono un certo numero di aggiustamenti: omissione dell'incontro di Francesca col poeta; sviluppo dettagliato di una materia narrativamente previa al flashback riportato da Dante; esegesi del matrimonio di Francesca e Gianciotto (Lanciotto in alcune versioni) come

3. Si ha notizia di: Francesca da Rimini - Ballo eroico in cinque atti (I825), composta da Vincenzo Schira e coreografata da Giovanni Calzerani e Giulio Viganò; Francesca da Rimini - Azione tragica in cinque atti (I838), comp. Saverio Mercadante, cor. Giacomo Serafini (questa Francesca da Rimini riprendeva con variazioni una Francesca d'Arimino - Azione tragica in 5 Atti coreografata dallo stesso Serafini nel I825); Francesca da Rimini-Opera ballo in quattro atti (I870), comp. Cesare Favetti, cor. ignoto; Francesca da Rimini-Azione tragico-mimica in cinque atti (I856), comp. Ferdinando Gualtieri, cor. Antonio Coppini. Cfr. "Francesca da Rimini: azione tragico-mimica in cinque atti: da rappresentarsi al teatro Apollo nel Carnevale I856 in 1857" (libretto dello spettacolo), Roma, Tipografia G. Menicanti, I856. Per dettagli ulteriori sulla Francesca da Rimini di Serafini-Mercadante, cfr. Regli 1838: 66-67. Per informazioni più dettagliate sulla fortuna di Dante nella musica del XIX secolo, cfr. Roglieri 200I: 74-IOO. 
ingiustizia ai danni di una fanciulla dalle forti predisposizioni sentimentali; biancheggiamento morale (e sessuale) dell'adulterio; apoteosi del legame nel ricongiungimento ultramondano degli amanti. Rettificazioni, tutte, che puntano a esaltare le possibili parentele poetiche tra Francesca e le sue omologhe nordiche, le Villi e Undine, redente da mille trasfigurazioni dell'amor profano in soffici Aldilà di nuvole e tulle.

Le tribolazioni ballettistiche di Francesca non sarebbero di nessun interesse se non illuminassero certi aspetti del dilemma - "teatralizzare il poema o "deteatralizzarlo" - che, negli adattamenti novecenteschi, i creatori dovranno invariabilmente affrontare. In un caso si tratterà di "inscenare" la Divina Commedia restituendone personaggi, scene e paesaggi, o di amplificarne la materia narrativa espandendone le digressioni; nell'altro di trasporre, del poema, il significato spirituale, la geometria, l'impianto dottrinario, il paradigma allegorico. Spenderlo nel valore sicuro della danza teatrale, o investirlo in una complessa negoziazione con gli assiomi poetici della danza astratta. Decidere a conti fatti se sia più opportuno, o più confacente al ruolo poetico della danza moderna, trattare la Commedia meno come la "storia" che come la "geografia" di qualcosa; comminarne l'interpretazione a un repertorio di "stati" più che di "atti"; di logiche, più che di discorso; e fare leva sulla capacità della danza di dispiegare il potenziale di anonimato e il "valore di cambio" (cioè la possibilità di trasposizione) di tutto quanto, nella Divina Commedia, cristallizza in nomi, personificazioni, prosopopee, "scene": universalizzandola, con il rischio implicito di renderla molto generica.

Ma poiché le opere maggiori (la Commedia è di quelle) tendono a scoraggiare la radicalità che per altri versi dovrebbero fomentare, è naturale che in settori (la danza è di quelli), avviliti da inveterati complessi d'inferiorità culturale, la difficoltà di "dire Dante" tenda a risolversi in un atteggiamento ibrido, parte narrativo, parte atmosferico, un poco spurio e prudenziale. Così, gesuiticamente fedeli al pregiudizio che la danza non possa valere se non come emozionante contorno alle pietanze forti di linguaggi più descrittivi, innumerevoli creatori sposeranno la prassi di far incarnare a un collettivo danzante $i$ dati "ambientali" dell'Oltremondo dantesco (una prassi a sua volta quasi imperativa negli adattamenti dichiaratamente teatrali del poema, che si sono di norma avvalsi della danza come di un "complemento" inesorabile): di esigere alla danza, come a una specie metereologia corriva, le opportune scorciatoie segniche, buone a dipingere oceanici sfondi di pena, penitenza o beatitudine.

Il paradosso di questo atteggiamento, che negozia la danza su un crinale sottile, tra il rischio di snaturarsi e quello di essere troppo genericamente se stessa, è che gli adattamenti più "infedeli" alla lettera dantesca potrebbero esserne in realtà le migliori captazioni intellettuali; le versioni più astratte potrebbero veicolare il nucleo semantico del poema con più efficienza che 
qualunque drammatizzazione, riduzione o trasposizione. Se il repertorio ottocentesco non lascia dubbi sulla strategia dominante, un'ambiguità di ben altro tenore si lascerà apprezzare, per la prima volta, nelle versioni "neoclassiche" della Commedia.

Francesca da Rimini (1937) di David Lichine, ${ }^{4}$ l'ultimo adattamento dalla Commedia che assomigli ancora a un balletto "ortodosso", può ritenersi in parte la prosecuzione inerziale di un modo ottocentesco di intendere l'adattamento, e in parte una reazione riflessa - o uno scongiuro - agli umori spirituali che nutrono la retorica della coeva danza moderna. $\grave{E}$ anche la più celebre, ma non l'ultima, ${ }^{5}$ delle messinscena realizzate dall'inizio del secolo ${ }^{6}$ nella scia dell'estetica diaghileviana, della Fantasia da Dante, Francesca da Rimini, op. 32 del 1876 di Pjotr Ilič Tchajkovskij (la musica più romantica di tutti i tempi, secondo Yuri Possokhov).

La Francesca di Lichine è un esempio squisito del pragmatismo che caratterizzava la compagine diaghileviana sin dall'era Massine: se da una parte ossequia le aspirazioni del neoclassicismo massiniano a una certa serietà filologica (Henry Clifford, storico dell'arte, collabora al libretto), dall'altra condivide con quel neoclassicismo la prassi calcolata di elevare il tasso di convenzionalità scenica e tecnica esorcizzando, in nome della stilizzazione e del colore storico, ogni rischio di deriva ideologica. Francesca da Rimini sarebbe insomma un ulteriore "Meta-balletto" dell'era massiniana, ${ }^{7}$ volto a celebrare il debito con i cliché e le esagerazioni sceniche del balletto teatrale di tradizione, e a incontrare le avanguardie plastiche e figurative solo in una ironia mimetica condivisa quanto ai valori di astrazione espressi paradossalmente dalla danse d'école. Allo sfoggiare le doti di colorismo immaginifico ed estenuazione carnevalesca associate alla fase terminale dei Ballets Russes, Lichine sembra voler occupare un quadrante estetico che le poetiche moderne, assetate di assolutismo e ritualità (o dedite a un brutto sogno di collusione con il totalitarismo), lasciano scoperto.

4. Lichine è coregrafo, in questo momento, dei Ballets Russes de Monte Carlo fondati nel 1932 dal colonnello Vasilij De Basil.

5. Un allestimento di gusto molto simile dello stesso balletto (su arrangiamenti di Liszt) sarà quello di Valrene Tweedie per l'australiano National Theatre Ballet nel 1955 (nello stesso anno Lichine riprenderà la sua versione per il Borovansky Ballet). Cfr. Garafola 20ıI; Pask, I982: 56.

6. Lo stesso Mikhail Fokine firma una Francesca da Rimini (1915) al ritorno in Russia dopo la separazione dalla compagnia di Diaghilev. Per informazioni dettagliate sulla struttura del balletto, cfr. Smith 1937: I9, e la libreta del Gran Teatro del Liceo, "Original Ballet Russe. Director: Col. W. de Basil", Temporada de Primavera 1948, disponibile nel sito. https://ddd. uab.cat/pub/societatliceu/societatliceupro/I948/42325-0I4@societatliceu.pdf.

7. Metaballettistica è altresì la scelta di trattare come musica da balletto un poema sinfonico come la Francesca da Rimini di Tchajkovskij, che non era nato a questo scopo, ma il cui adattamento a un'impresa teatrale si giustificava nell'ottica della strategia ideologica dei Ballets Russes imperniata sul trattamento di Tchajkovskij stesso come emblema assoluto della cultura del balletto imperiale. 
E giacché si tratta di rilanciare i modelli romantici e di superfetarli, Lichine non esita, sulla scorta dei rimaneggiamenti di Gabriele D'Annunzio e Riccardo Zandonai, ${ }^{8}$ a rimaneggiare disinvoltamente sia il racconto tradizionale che il programma Tchaikovskiano (che contempla almeno un prologo nella selva oscura e un'impressione sinfonica della bufera infernale): un Gianciotto deforme avrebbe mandato a innamorare Francesca e ottenerne la mano il più avvenente Paolo, suo fratello, solo per prendere il posto al momento delle nozze. L'infelicità del matrimonio risospinge Francesca tra le braccia di Paolo. Dopo un interludio danzato sui casi di Lancillotto e Ginevra, e un duetto d'amore tra i protagonisti, Paolo sfida Gianciotto a duello e ne viene ucciso (il libretto sposa senza ambagi il pruriginoso fraintendimento che interpreta il verso "Quel giorno più non vi leggemmo innante" come l'irruzione del cornuto accorso a pizzicare e uccidere gli adulteri in piena lettura). L'eroina si lascia cadere sulla spada di cui è morto l'amato. Arricchiscono la trama le manovre complementari di una suivante compassionevole, Chiara, e del delatore Girolamo (riedizioni di Emilia e Iago).

Gli stuoli di cortigiani perversi e nani infernali (coadiutori delle malefatte di Gianciotto), e di emissari celestiali (pronubi alle effusioni dei due amanti giacché, grazie all'amore, heaven is a place on earth), sono ciò che resta del tentativo di Lichine di conciliare il rimpolpamento a tinte forti dell'allestimento con una pallida restituzione del dualismo dantesco (inferno-paradiso, abiezione-perfezione), non senza avallarne la simmetria con il dualismo a sua volta più usitato nella legittimazione ideologica del Neoclassicismo: lo scontro tra una realtà segnata dall'ingiusto trionfo della deformità (Gianciotto verrebbe così a essere il laido rappresentante di tutti gli espressionismi del secolo e l'incarnazione vituperevole dei suoi materialismi), e il Balletto stesso, assurto sin dalla Morte del Cigno (1907) a codice di trasfigurazione, ricettacolo terminale di una bellezza troppo perfetta per questo mondo: forma, tout court, di un'angelismo che alle reviviscenze della tecnica classica, alle sue stilizzazioni e "ascensioni", fa incarnare tutti i Paradisi perduti che verranno.

Lichine è profetico: non solo della svenevolezza con cui, nel settore neoclassico, si affronterà d'ora in avanti la rappresentazione del Paradiso dantesco (il Neoclassicismo se la vedrà un po' peggio con i regni inferiori, per comprensibili ragioni di incompatibilità stilistica), ma anche della tentazione diffusa, persino in ambito moderno e contemporanei, di ricorrere al balletto e alla sua "differenza qualitativa” per rappresentare il tono dell'ultima Cantica. Nel bel

8. La tragedia Francesca da Rimini di Gabriele D’Annunzio è del I902. L’opera omonima di Riccardo Zandonai, su libretto di Tito Ricordi, è del I9I4. Benché si dichiarassero ispirate a Inferno $\mathrm{V}$, ambedue le versioni evitavano di drammatizzare l'incontro tra Dante e Francesca nel contesto del cerchio degli incontinenti, per poter articolare liberamente un intrigo completamente terreno di passioni e delitti d'epoca, lasciandosi semmai ispirare parzialmente dal poema di Leigh Hunt Historia de Rimini (I816; ed. Johnson I89I). 
mezzo di versioni totalmente contemporanee, non sarà infrequente vedere le Beatrici tardo-moderne profondere beatitudine dall'alto di scarpette a punta piuttosto incongruenti. E giacché la lettura amorosa sarà, a tutte le latitudini dello stile, la più gettonata delle scorciatoie esegetiche e prevarrà la norma di leggere la Commedia come un romanzo sentimentale, verrà spontaneo fare di una Beatrice "classicheggiante" l'incarnazione di una nostalgia che, a conti fatti, comporta non poche insinuazioni sull'innocenza strutturale e regressiva, sul candore paradisiaco del Balletto come genere tout court. Se Beatrice è insomma erede di Francesca è perché la Francesca di Lichine, astutamente candeggiata da un Neoclassicismo di matrice diaghileviana, è già, a sua volta, erede di Beatrice: l'irruzione postuma di una "santità" del movimento che non ha quasi posto nell'inferno dei tempi moderni. ${ }^{9}$

Esistono dunque due parametri suscettibili di trasformare in un dilemma formale l'approssimazione neoclassica alla materia dantesca: la narratività (il timore che l'estensione oceanica e la sostanza morale del poema invochino un trattamento meno drammatico - ma chissà se più "drastico" - di quello approntato finora dal balletto teatrale) e l'erotismo (il timore che la tensione erotica del poema - tradizionalmente sussunta nel dualismo demenziale tra la passione tanatica di Paolo e Francesca e l'amore salvifico di Dante e Beatrice - debba attenuarsi per fare spazio a turgori più propriamente morali, sociali o religiosi). In un momento in cui il balletto occidentale accetta la sfida di competere con la danza moderna sul terreno di una rinuncia comune all'ingenuità, il problema-Dante è una buona sineddoche delle tensioni generali da cui, negli anni ' 40 , scaturirà l'ipotesi del Neoclassicismo astratto.

\section{DANTE: QUASI UNA SINFONIA DANZATA}

La nozione di Symphonic Ballet è di fatto un compromesso: il settore di balletto britannico che la conia non rinuncia a intendere l'esenzione da imperativi narrativi - implicita nella messinscena "astratta" delle partiture sinfoniche - come una licenza a interpretare allegoricamente le implicazioni morali o concettuali della musica. I "personaggi" del vecchio balletto si vedranno il più delle volte surrogati dalle "personificazioni" del nuovo. E poiché l'approccio in questione suole ispirare dicotomie spicce e manicheismi facili, è comprensibile che la

9. Nella genealogia succinta delle Francesca da Rimini posteriori, più vicine, almeno sulla carta, all'idea di recuperare parzialmente il dettato di Dante, e dunque di rappresentare nuovamente la dimensione ultramondana del legame tra Paolo e Francesca, il risultato sarà di rappresentare l'Inferno stesso in toni molto sublimati. È il caso della Francesca da Rimini (20I2) firmata da Yuri Possokhov per il San Francisco Ballet e tuttora in repertorio, dove persino le tre "guardiane dell'Inferno" introdotte da Possokhov per dare il tono della prima Cantica, non sono più temibili, né più credibilmente infernali, che tre Grazie vestite di rosso. Cfr. Tsao 2013. 
tensione tra istanze allegoriche opposte tenda ad assumere la forma riconoscibile di pas de deux conflittivamente erotico, che è una prerogativa spiccata del Neoclassicismo britannico: l'allegoresi ballettistica fomenta a conti fatti un'impensabile conciliazione tra astrazione ed erotismo, tra morality religiosa e aneddoto profano, tra agon e eros, che sarà il piatto forte della poetica di Ninette de Valois, Frederick Ashton e, in misura minore, Antony Tudor, Agnes de Mille o lo stesso Balanchine.

Dante Sonata (1940) ${ }^{\mathrm{IO}}$ di Ashton afferisce a questa mésalliance. Sensibile a quello che crede il tema centrale della Commedia - la lotta tra Bene e Male -, probabilmente condizionato dall'oscurità della congiuntura storica (il I940 è un'annata di trionfi hitleriani), e scoraggiato dai grandi numeri della Commedia a farne una sintesi propriamente narrativa, il coreografo britannico finisce per considerare la lettura allegorica il più accettabile dei compromessi. Secondo un dualismo già brevettato da de Valois in Job (193I) o Checkmate (1937), Ashton divide il corpo di ballo in due ranghi - Children of Light (in bianco) e Children of Darkness (in nero, questi ultimi, e con serpenti avvolti al corpo) - i cui corifei possano altresì, nei numeri solistici e a due, incarnare personaggi storici o mitici, rappresentativi di precise forze morali.

Sospeso tra allegoresi corriva e teatralizzazione esplicita del referente letterario, La Divine Comédie (1976) ${ }^{\mathrm{II}}$ di Vittorio Biagi (ex ballerino del Ballet du XXe Siècle) è in un certo senso l'ultima delle versioni di Dante ancora immuni - per il fatto di adottare un linguaggio ballettistico $-{ }^{\mathrm{I} 2}$ alla tentazione laica di decostruire la fonte o di dissacrarla. Biagi opta dunque per "secolarizzarla" o, più precisamente, applicarla alla sacralizzazione forzata di alcuni temi secolari. L'insistenza della Commedia di Biagi su temi carnali è in fondo un retaggio di Maurice Béjart, il coreografo più attivo di tutti i tempi in materia di trasfigurazioni pseudo-mistiche della vita pulsionale. Pure, è proprio questa deformazione (la superfetazione teologica di temi corporei) l'aspetto più intrigante de La Divine Comédie, se valgono le dichiarazioni di Parmegiani sulla committenza musicale del balletto: "Être en enfer, c'est être contraint de vivre la tête en bas - selon une vision naïve des antipodes - et penser que l'Enfer

IO. Per una descrizione più dettagliata del balletto, cfr. Zicari 20I3; Everson 2007: 53-64.

II. Prod. Ballet de Lyon, creaz. 6 Ottobre 1976, Opéra de Lyon.

I2. Non deve ingannare in questo senso né la scelta di un accompagnamento musicale schiettamente moderno (Enfer di Bernard Parmegiani). Biagi fu ballerino di Maurice Béjart, la cui lealtà alla corrente della musica concreta è nota sin dai tempi di Symphonie pour un homme seul. Enfer (197I-1972), composizione elettroacustica con voce accompagnante di Michel Hermon (espunta nelle versioni successive della stessa partitura), è definita dallo stesso autore, decano della musica sperimentale francese, come un "cantique électronique". In anni successivi Parmegiani procede con la collaborazione di François Bayle alla composizione di un Purgatoire e di un Paradis che completeranno la trilogia, probabilmente nella prospettiva di una parallela estensione degli allestimenti coreografici. Tuttavia Vittorio Biagi non andrà oltre la messinscena danzata della prima parte. 
égale l'Envers. Le monde s'y trouve inversé, on s'y enfonce tel une toupie dont la pointe est attirée vers un centre profondément enfoui [...]. Dans notre chair il existe de ces points [...]." ${ }^{3}$

Facendo della Commedia un apologo laico intorno all'abissalità di un corpo cui Béjart aveva da sempre comminato virtù superomistiche, e celebrando in tutte le salse il potenziale di dissidenza di sesso e natura, anche Biagi evita con cura la tentazione di narrare il poema. E costruisce la danza (nella versione erotico-eroica di neoclassicismo collaudata dal Ballet du XXe Siècle) su una gamma di contraintes (costrizioni) - sette come i peccati capitali - chiamate a catalizzare nel corpo danzante fenomeni di inversione, resistenza o addirittura per-versione che sono il correlativo danzistico dell'equazione EnferEnvers invocata da Parmegiani (riproponendo l'uso polemico degli en dedans e enveloppés cari a Béjart). Di là dagli inevitabili manierismi, si può riconoscere a Biagi di essere stato il primo (e per molto tempo il solo) ad ammettere che la sostanza cinetica dell'Inferno fosse da ricercarsi nella "frizione": una turbolenza di desiderio e costrizione, di arbitrio e coazione. ${ }^{\mathrm{I} 4}$

Leggere la prima Cantica come sede astratta e cinetica di un avvitamento della contradditorietà umana comportava una rilettura complessiva dell'Inferno come indice generale della saturazione di un corpo affollato di desideri, resistenze, reticenze, castighi, pulsioni, contraddizioni - "dia-bolico" nel vero senso della parola, perché irretito nel gioco dei suoi antagonismi. L'idea semplicissima dell'Inferno come castigo autoinflitto della carne invece che come sede di rappresaglie morali è forse il più misconosciuto contributo del Neoclassicismo al dibattito implicito sul senso della Divina Commedia: per decenni, e sostanzialmente fino alla trilogia di Emio Greco (di cui tratterò oltre) non se ne aggiorneranno le implicazioni poetiche.

\section{DANTE: QUASI UNA PERDONANZA}

Se è vero che le Commedie danzate degli anni '9o sono poche e artisticamente poco significative, è anche vero che tutte riflettono, con un tono tra sacrale e sentimentale, l'enorme successo, negli stessi anni, di alcuni dei valori che stanno strutturando le idolatrie della postmodernità: sincretismo New Age con note di spiritualismo prêt-à-porter; passione acritica per la multimedialità come apertura speranzosa (non già inflazione disperata) dei linguaggi; spirito di conciliazione universale, non esente da allusioni sublimi all'Eterno

I3. Dichiarazione di Parmegiani, cit. in Ressources. IRCAM: brahms.ircam.fr/works/work/36879.

I4. In questo senso è anche da interpretarsi, nel Purgatoire di Bayle, l'idea di un Ange Feu, omaggio implicito a quell'Angelo di Fuoco che, nell'opera omonima di Prokofiev (I919), già aveva rappresentato un'allegoria del desiderio sessuale come sintesi si dannazione e redenzione. 
Femminino e ai suoi poteri. In questo clima di revival dianoetico e beatitudini laiche, Beatrice ha tutti i titoli per divenire la patrona delle esegesi express. E poiché la relazione della prassi scenica con Dante s'impronta anche in danza a usi sempre meno cerimoniali e sempre più cerimoniosi, le produzioni inerenti si concepiranno come realizzazioni di un certo respiro spettacolare, affidate alla mano sicura di creatori celebri la cui verve di trasposizione sia abbastanza blanda da non compromettere il tono elevato della proposta, o così abituati a risolvere "carismaticamente" gli antagonismi stilistici presenti nel loro linguaggio da applicare le stesse scorciatoie carismatiche alla rimozione del problema ermeneutico e morale che comporta il poema dantesco.

Affrontato in questi termini, spirituali (o sospettosamente mistici) senza essere confessionali, e bagnato nelle calde lacrime di una religiosità vagamente materna, Dante sembra pronto a benedire, perdonandole tutti i suoi antagonismi, la danza che lo benedice. Se nella danza dei primi anni '9o vi sono faglie in cui si gesta il sisma destinato a sommuovere tutta la sua geografia poetica, questi incontri conciliatori e sincretici con l'universo dantesco vegliano a che le stesse crepe e divisioni appaiano come valori aggiunti: spacciano le rimozioni per promozioni. Dante Symphony (1990) ${ }^{15}$ del belga Micha Van Hoecke e Commedia (1993) ${ }^{16}$ della finlandese Carolyn Carlson lavorano in questa direzione.

Vero e proprio pezzo d'occasione, ${ }^{17}$ lo spettacolo di Van Hoecke, coreografato sull'omonima sinfonia programmatica (1857) di Liszt, e sulla Fantasia quasi Sonata per pianoforte Après une lecture de Dante (1849) dello stesso compositore, si annuncia come un "poema coreografico": definizione che, invocando la presunta facoltà della danza di riassumere stati emotivi e condizioni spirituali, da una parte aggira sportivamente il problema della vastità del poema dantesco, dall'altra esenta il coreografo dalla necessità di allegoresi spinta che ha segnato le letture neoclassiche viste sin qui. L'assioma di Van Hoecke è che la sintesi sia privilegio dei linguaggi universali (e che la danza sia di quelli). Così, con uno stile eclettico, che unisce neoclassicismo bejartiano, influssi etnici ed elementi di Tanztheater, van Hoecke opta per rimaneggiare rispettosamente la fonte dantesca in blocchi separati: isolando all'inizio dello spettaco-

15. Prod. Ravenna Festival, Festival della Riviera Etrusca Castello Pasquini, creaz. I9 agosto I990, Rocca Brancaleone, Ravenna. Per una descrizione più dettagliata del balletto cfr. Testa I990.

I6. Prod. Schauspielhaus Hamburg, creaz. I5 marzo 1993, Amburgo. Cfr.: https://www.numeridanse.tv/fr/video/2045 commedia.

17. Dante Simphony viene creato espressamente per la Rocca Brancaleone, uno dei luoghi emblematici della storia cittadina, in apertura del Festival di Ravenna (che ha in questi anni, sotto la direzione di Cristina Muti, una vocazione eminentemente musicale) e con un esplicito intento municipal-encomiastico, in considerazione del ruolo simbolico di Ravenna come luogo di sepoltura di Dante. Nel balletto gli spalti della Rocca sono altresì percorsi dai danzatori all'inizio dell'ultima sezione. 
lo l'epigrafe di testo introduttiva (l'incipit della Commedia recitato da Franco di Francescantonio); percorrendo quindi con risultati estetici discontinui le tre Cantiche; quadrando il lavoro sull'asse erotico tra le due coppie, sublimatainnocente (Dante e Beatrice bambini) e tragico-sensuale (Paolo e Francesca, immancabilmente); ma soprattutto sceverando con innegabile fiuto scenico ciò che è teatralizzabile (episodi e personaggi identificabili) da ciò che non può rappresentarsi se non in termini generali (climi, sensazioni, stati morali).

Van Hoecke trasforma il potenziale malessere del suo sincretismo (una convivenza forzata di astrazione e teatralità) nel vero atout estetico di Dante Symphony: inventa un cubo di cristallo - totem dell'allestimento e centro di differenti operazioni plastiche, tra cui le violente colate di vernici che rappresentano il clima dell'Inferno come una cupa testura di colori - per straniare i differenti "pacchetti d'immagine" che segnalano il passaggio da un livello all'altro dell'Oltremondo, e poter riservare alla danza lo spazio vuoto attorno a questo "modulo". Ma soprattutto decide di assegnare a ogni cantica, senza troppi scrupoli critico-ideologici, una differente temperatura stilistica: dipingendo l'Inferno come un luogo "astratto", convulso, a volte acrobatico; il Purgatorio come un "Teatro-Danza" intessuto di gesti quotidiani; e il cielo dei beati come un elisio minimalista di gestualità estatica, intrisa di elementi orientali (come il lieve ondeggiare della testa dei danzatori che avanzano in meditazione, sulle voci bianche del Magnificat).

Operazione di segno analogo, due anni dopo, è Commedia ${ }^{18}$ di Carolyn Carlson. A cominciare dal trattamento omeopatico della questione testuale: esemplari della Commedia sono religiosamente aperti a proscenio, mentre una specie di florilegio dantesco si dà negli interludi dei "dicitori" che recitano passaggi dal poema in varie lingue - in ossequio alla famigerata "universalita" del testo dantesco -; le musiche (sotto la supervisione di René Aubry e Michel Portal) sono un astuto centone di motivi etnici ed elettronici; il tenore estetico (scene e costumi) dello spettacolo sembra viziato da un gusto leggermente kitsch ed ellenizzante che pretende sottolineare l'intemporalità del dettato dantesco. Carlson amplifica il generalismo che Van Hoecke conseguiva ancora tenere a bada con stratagemmi plastici: mentre il cubo di cristallo del belga era ancora, in un certo senso un modulo di "contenzione" degli eccessi numerici del poema, i globi dorati di Commedia - quasi una replica a quel cubo, fedeli al costume imbelle di arredare Dante con ogni tipo di succedaneo tolemaico - esprimono in ogni momento una pretesa un po' bamboleggiante di cosmicità. ${ }^{19}$

I8. Per una descrizione più dettagliata di Commedia, cfr. Carlson 200I; Turnbull 1999; Delcourt 2015 .

19. Sembra impossibile mettere in scena la Divina Commedia senza cercare di evocare la sua universalità e cosmicità attraverso scorciatoie sferologiche (una grande sfera dal cui retro 
Dall'egemonia scenica della sua Beatrice (rossovestita e bendata per dare forma al tono erotico e giustiziere della sua entrata in Purgatorio $X X X)$, può dedursi che Carlson intenda interpretare il poema come un episodio nella storia mistica dell'Eterno Femminino, o Ewigweiblichkeit, e un capitolo ulteriore della saga (s)olistica da lei iniziata con Blue Lady (1984); $;^{20}$ davanti a questa solennizzazione uterina della metafisica dantesca, e in assenza del benché minimo incidente ermeneutico, si capisce che parte della critica azzardi un parallelismo tra la poetica dell'operazione carlsoniana e l'ipotesi teorica di Guglielmo Gorni, che in Dante legge l'invocazione di uno spirito avvolgente e misericordioso, di una totalizzazione che aspetta di compiersi nella realizzazione della cupola - stilema femminile per antonomasia - di Santa Maria del Fiore. ${ }^{2 I}$

Nel nucleo della "concentricità" di cui si alimenta la poetica di Carlson lavora una benevolenza simile a quella che, negli stessi anni, avvicina una parte dei Dance Studies alle ricette confortanti del Transmodernismo, ${ }^{22}$ l'interpretazione del tempo presente (il muro è appena caduto) come superamento ed entelechia della modernità nell'estinzione ecumenica del conflitto con le alterità, nella conciliazione definitiva di centro e periferie. Di qui a poco, in settori accademici refrattari al pessimismo, la buona novella trasmoderna sposerà certi aspetti del Womanismo ${ }^{23}$ di Alice Walker (Walker 20II.) per partorire la teoria danzistica più paradisiaca di tutti i tempi: la theodancecology, ${ }^{24}$ volenteroso riciclaggio body-mind della Performance Theory precoce. ${ }^{25}$ Prodromo alla sfilza di pseudo-paradisi che punteggerà questo studio, il concetto transmoderno e womanista di self-actualization, così simile all'equilibrismo mistico, nella poetica di Carlson, tra donna-come-totalità e totalità-comedonna, può paradossalmente ricordare l'autorealizzazione delle schiere celesti descritta da Dante: come coscienza a tutto tondo, "olografica" e risolutamente consensuale, è davvero il candore terminale di una Candida Rosa. ${ }^{26}$

emergono gli interpreti si ritroverà, insieme a una grande spirale di rame discendente, anche nella polemica messinscena di Eimuntas Nekrošius del I9I2. Globi dello stesso tipo torneranno nelle versioni coreografiche, tra gli altri, di Emio Greco (v. oltre).

2o. Titoli della stessa sequenza di lavori dedicati a declinare l'essenza dinamica e spirituale della femminilità come nuovo assoluto post-cristiano sono Maa (1991), Vu d'ici (1996), Inanna (2006), Pneuma (2014) e altri.

2I. Cfr. Gorni 20II; Wittschier 2004: 58 segg.

22. Mi rifaccio all'insieme di tesi sulla storia della cultura elaborato da Enrique Dussel (1996).

23. Cfr. Walker 2oII.

24. "Thus, I define Theodancecology as a womanist transmodern field that comprises the study of the sincretic power and assets contained in dance in ontological knowledge production and dissemination through mystical meaning and experiences" (Walter 20I4: 39-57).

25. "His [sc. the performer's] entire effort is in making his body-voice-mind-spirit whole" (Schechner 1977: 57).

26. Non è peraltro casuale che, a differenza di approcci più politici o sociologici (che tendono a svincolare la danza dalla musica per "emanciparla"), la parte di womanismo associata alla 
Afferenti, anche cronologicamente, alle tendenze sincretiche della postmodernità matura, le versioni di Van Hoecke e Carlson sono dopotutto un corollario poetico alla famiglia degli adattamenti - liberi anch'essi da eccessive inquietudini esegetiche - creati nella cornice del gusto neoclassico. Il loro eclettismo stilistico è estraneo allo spirito di decostruzione scrupolosa o di trasposizione cinica che influenzerà gli adattamenti dello stesso decennio e del successivo. Van Hoecke, venuto dal Neoclassicismo, ha cercato di elaborare con mezzi empirici una versione assai idiosincratica di modernità. Viceversa Carlson, rappresentante di spicco dell'ultima Modern Dance, è chi con più ostinazione ha lavorato, negli anni '9o, a canalizzare le asprezze dello stile moderno in una prassi abbastanza "grafica" e confortante da potersi leggere come una classicità di secondo livello. Ambedue sono personalità di transizione, non solo perché sembrano determinati a superare empiricamente (Van Hoecke) e ideologicamente (Carlson) l'antagonismo già anacronistico tra classicità e modernità, ma perché questa soluzione di un falso problema permette loro da una parte di mantenersi in equilibrio tra una sconfinata fiducia nei poteri di universalizzazione e "arrotondamento" della danza, e l'angoloso panorama di disequilibri di una danza coeva che minaccia il prestigio di entrambi; dall'altra, di ritardare, anziché fomentarlo, il processo di de-teologizzazione della Commedia (Barolini 1992; Wittschier 2004) che sarà il tratto più genuino della messinscena post-moderna.

\section{DANTE: QUASI UN'ANIMAZIONE}

Nei lavori di Van Hoecke e Carlson, l'eclettismo vago delle soluzioni, il muoversi a tentoni tra danza, acrobazia, mimo, declamazione e action painting, prelude a che le negoziazioni degli anni ' 90 tra le arti performative e il multiverso dantesco si sviluppino almeno in parte attorno al motivo della multimedialità, meridiano dell'agopuntura culturale del decennio. Da queste negoziazioni emergerà un ceppo specifico e frondoso di adattamenti, anche recentissimi, della Commedia.

L'insistenza sul fatto che il poema sia, per la sua sensazionale ricchezza d'immagini, per la coralità spiccata e le arditezze spaziali, un testo multimedia ante-litteram è una banalità esegetica e un modo sbrigativo di ammettere l'impotenza della danza sola a significare Dante; il sintomo, ancora, di una

danza rivendichi, argomentandola storicamente, l'interdipendenza ancestrale e finanche l'equazione "olografica" o sinestetica di danza e musica (il Paradiso è anche la Cantica in cui si realizza alla lettera la vecchia utopia balanchiniana di sentire la danza e vedere la musica). Non è un caso che proprio Walter insista sul paradigma della danza sociale afroamericana, da un punto di vista teodanzecologico, come "rejoicing in reflection of God" (vera e propria riedizione gospel del paradosso consensuale che Dante riassume nel verso, "che quel vuole Iddio e noi volemo" (Pd. XX, I38). 
rinuncia a rintracciare un senso della Commedia per la danza occidentale nel cuore stesso della sua identità squisitamente letteraria; e un sintomo dell'irrinunciabile luogo comune che ha autorizzato, nel primo decennio di questo secolo, l'epidemia di classici adattati televisivamente ai bambini di tutte le età anagrafiche.

Il risultato è una nuova mésalliance sistematica: per non dover abdicare alla possibilità di iscrivere Dante nel registro delle arti vive, danza e teatro delegheranno alla multimedialità il compito di dispiegare tutta la gamma di rappresentazioni, analogiche o trasposte, della materia del poema, circondando di "proiezioni" più o meno critiche, più o meno criptiche, la sostanza cinetica di una performance o di una danza troppo generiche per apparire dovutamente dantesche.

Trascorso il periodo di auge della video-arte e della video-danza, ricco in avventure anche egregie di adattamento spregiudicato della lettera dantesca ai nuovi linguaggi visivi, la danza teatrale a cavallo tra vecchio e nuovo millennio non sembra ricorrere alle nuove tecnologie che per supplire a un deficit di lungimiranza esegetica.

Un poco intossicata dalla convinzione che la sua missione sia da una parte di incarnare dinamicamente le "sensazioni" morali del poema, dall'altra di commentarlo con complementi sensoriali alternativi, la danza del periodo pare destinata a istituire attorno a Dante una forma paradossale di metadiscorso, in cui ambedue, danza e immagine, l'una troppo generica, l'altra troppo connotata, si tengono rigorosamente, come glosse misteriose e commenti a mezza bocca, ai margini di una sostanza poetica sempre prorogabile, sempre un po' sfuggente: l'Oltremondo si diluirebbe in una pioggia di associazioni cinetiche o visive se non se ne confidasse la declamazione a un attore, in una specie di resa incondizionata alle funzioni didascaliche del testo. Operazione meno univoca di quanto appaia, poiché quand'anche lo spettacolo si presentasse come un intreccio di segni che rimandano perifericamente a luoghi specifici del testo, il dettato potrebbe non esprimersi in citazioni abbastanza dirette da far quadrare senza scarti tutti i segni che l'accompagnano: il più delle volte $\mathrm{i}$ versi della Commedia potrebbero apparire dissociati dal loro contesto letterario e risultare, a conti fatti, un "commento" enigmatico al commento su Dante, al collage o labirinto metadiscorsivo che lo spettacolo è divenuto. ${ }^{27}$

Sono innumerevoli, anche in anni recenti, gli adattamenti di questo tipo, tendenti a trasformare la danza nella postilla espressiva, tutto sommato inter-

27. Accade qualcosa di simile in Dante's Divine Comedy (prod. Internationl Fringe Festival, 2009) di Rene Migliaccio, direttore della newyorkina Blackmoon Theatre Company. Qui il performer Alessio Bordoni alterna movimento e declamazione di frammenti sparsi della Commedia, in una scenografia multimediale avvolgente fatta di retroproiezioni dei videocollages animati dell'artista India Evans. 
cambiabile, di una suite d'immagini che valgono a loro volta come postilla intercambiabile alle danze. La Divina Commedia si dissolve in una specie di diaframma ineffabile, l'interstizio postulabile di un'intertestualità ipertrofica. Questa circolazione dei rimandi - tra una danza prodotta per dissociazione, e un'immagine prodotta per associazione - è tutto ciò che resta del periplo dantesco e delle sue proprie dinamiche di circolazione, del suo proprio schema di ritorni spaziali e ridondanze intertestuali.

Versioni "deboli" in tal senso sono quella di Thomas Goritzki (Die göttliche Komödie. Ein Spektakel aus Schauspiel, Tanz und Musiktheater nach Dante Alighieri, creaz. Stadttheater Giessen, 2008) ${ }^{28}$ e quella, su cui mi soffermerò brevemente, firmata da Keith Ferrone per la Florence Dance Company (da lui fondata con Marga Nativo): Divina.com. From Here to Eternity (201I). ${ }^{29}$

Creata per onorare un incarico municipale, su musiche di Maurizio Fasolo e Enzo Regi dell'ensemble di Transavanguardia elettronica Pankow (in collaborazione col pianista compositore Stefano Maurizi), Divina.com non ha niente a che vedere con l'universo di telepresenza che il titolo suggerisce, e non allude all'erranza peculiare che è la navigazione in rete se non per promuovere lo scambio, la ".comunicazione" quasi randomica di segni tra danza moderna e immagine astratta che innerva l'operazione. Come in una specie di event cunninghamiano fuori tempo massimo, la proiezione e animazione su pannelli semoventi della serie di pitture astratte The Ascent of Orpheus: Between and Beyond Representation and Abstraction dell'americano Adi Da Samraj, invitato del Bargello, fa da sfondo, qui a tutte le danze della Florence Dance Company.

Si pretende che i motivi astratti e colori primari di quest'opera, memore dell'universo formale di Miró, evochino "the great struggle to achieve divine order amidst the apparently hellish maze of experience", ${ }^{30}$ e che in questo senso l'automozione delle immagini possa fare eco alla mozione dei corpi in cerca di un equilibrio definitivo. Forse persuasi, con argomenti che ricordano il suprematismo russo, dell'intrinseca divinità e della vocazione essenzialmente cinetica delle forme pure, Ferrone e Samraj sottomettono tutta la loro lettura del poema a una fede incrollabile nell'eloquenza simbolica dei colori primari (rossi e gialli, come no, per l'Inferno; bianchi a azzurri, come no, per il Paradiso), nel valore psico-descrittivo delle linee (ascendenti, discendenti, spezzate) e nel carisma semantico degli emblemi (le scale e le sedie, nel Purgatorio, come simboli di ascesi e attesa, o il cerchio bianco in campo azzurro che rende con

28. Cfr. Gumts 2008.

29. Prod. Museo del Bargello, Florence Dance Company, Adi Da Foundation, Ministero per i Beni e le Attività Culturali e del Tursimo; creaz. 9 luglio 20ı,, Museo del Bargello, Firenze. Lo spettacolo sarà ripreso nel 20I5 in occasione delle celebrazioni per i 750 anni della nascita di Dante (dello stesso anno sarà il Ballo I265 di Virgilio Sieni, analizzato più oltre).

30. Adi Da Samraj, cit. nel dossier Adi Da and Adidam: News and Personal Pespectives, dossier online dell'espozione al Bargello: http://www.theascentoforpheus.org/. 
compiaciuto minimalismo la visione di Dio di Paradiso XXXIII, tra palloni e orbi cristallini, per cambiare). La danza sarebbe a sua volta piuttosto generica (Ferrone è pur sempre artista di ascendenza neoclassica), non fosse che nel Paradiso torna inesorabile il lavoro sulle punte ad avallare una credenza dura a morire sul balletto come segno di trascendenza e indeterminazione. Nella stessa sezione, in linea con l'ecologismo dello Zeitgeist post-moderno, la compagnia completa il quadro di gioie romite e ultramondane creando in tempo reale un giardinetto di fiori in vaso, che sullo sfondo del cerchio bianco di Samraj sembra un'ode all'alleanza tra natura e astrazione.

Ciò che non si vuole ammettere è che, se davvero si tratta di trascendere Dante e le sue trascendenze come una precessione compassata di forme astratte e diagrammi di pensiero, o viceversa di desumere dal poema una cartografia cangiante di pure intensità; se la sfida è traslare la pluralità della visione dantesca a una peripezia di cose e forme (torturate nell'Inferno, estenuate nel Purgatorio, sublimate nel Paradiso), la sola danza plausibile per la Commedia è quella che all'animazione - digitale o tradizionale - delega senz'altro il compito di raccontare Dante come una precessione astratta di fenomeni grafici e regimi materici, concrezioni e cristallizzazioni. ${ }^{3 \mathrm{I}}$

La profusione dei corredi audiovisuali negli adattamenti danteschi del primo scorcio di secolo tradisce alcuni malesseri oggettivi: il primo è la difficoltà pura e semplice, per la danza, di contenere ed esporre la massa narrativa del poema (funzione che è stata frattanto accaparrata dai formati commerciali che analizzerò più avanti); il secondo attiene alla difficoltà di rappresentare persuasivamente, con gli organici modesti di una compagnia di danza (debole di fronte ai grandi mezzi dei generi mainstream), le sue "masse". Ne consegue la convinzione crescente che l'approccio danzato al poema non potrà, per limiti materiali, che essere singolare e meditativo (parlerò oltre dei Dante "da camera" debitori anch'essi di questa logica). Che si possa trasformare la Commedia, convenevolmente, in ciò che dopotutto è sempre stata: l'esperienza mentale di un soggetto; e sfruttare le tecnologie digitali per restituire il tasso di astrazione di una peripezia tutta intellettuale e percettiva. Al filone multimediale degli adattamenti dal poema non mancheranno esemplari suggestivi

3I. Di là dal memorabile TV Dante. Cantos I-VIII di Peter Greenaway per Channel 4 (London, Argos Films - British Film Institute, I995; Digital Classic, 20II), è il caso di ricordare, in una linea di lavoro che sta tra la performance e l'installazione, negli anni ' 90 , lavori come Electronic Purgatory. Eine digitale Musik-Theater-Komposition für Schauspieler di Kristi Allik e Robert Mulder (Bourges, I989; cfr. Allik-Mulder 199I); Zwischen Himmel und Hölle. Eine multimediale Inszenierung nach Dante Alighieri di Wolfram Lensen (Zweckel, Alte Maschinenhalle, 1999); The Dante Project: Inferno, diretto da Bill Conte e presentato da Synchronicity Space (New York, Mercer Street, 1996). E ancora Reading Dante di Joan Jonas, ciclo di collages multimedia e video-performances (2008-2010), e la videoanimazione Divine Comédie di Simon Coté-Lapointe (20I4); cfr. il sito http://simoncotelapointe. com/?page id=2. 
di questa categoria "monodrammatica" di lectura dantis. ${ }^{32}$ Poiché insomma la parte di poetica che concepisce come un problema d'immagine l'attualizzazione della Commedia non contempla la sua ascesi definitiva, il "Paradiso della danza", se non come gesto paradossale di smaterializzazione e trasumanazione visiva della danza stessa e del corpo che la fa, le approssimazioni più eloquenti e più inopinatamente fedeli alla fenomenologia del Paradiso apparterranno, per gli ultimi dieci anni, al settore della performance digitale. ${ }^{33}$ Penso al lavoro della compagine halflangel diretta da Jools Gilson-Ellis e Richard Povall, specializzata nell'allestire intorni elettronici o intelligent stages in cui è il movimento degli interpreti a creare lo spazio sonoro e luminotecnico in cui si muovono. Ė il caso di Spinstren (2002), grande giostra di aneddoti, moves, miti e racconti attorno al concetto di rotazione. I versi in cui i beati proclamano in coro la simmetria di volizione che regge il dispositivo "glorioso" del Paradiso trova un'eco sorprendente nella retorica che questa e altre compagini hanno organizzato attorno all'idea di spazio digitale interattivo: "The sensation of gesture instantaneously effecting sound, lights and projections creates a heightened sense of presence, of being in the body in an enlivened state. The space takes on a quality of consciousness as the dancer extends his/her volition into

32. È una versione digital-performativa di questo tipo la video-installazione di Marco Donnarumma Hypo Chrysos (HC) (20I4). Cfr. Donnarumma 20I2. Mi rifaccio per la descrizione alle parole dell'autore stesso: "Hypo Chrysos (HP) is a work of action art for vexed body and biophysical media. During this twenty minutes action I pull two concrete blocks in a circle. My motion is oppressively constant. I have to force myself into accepting the pain until the action is ended. The increasing strain of my corporeal tissues produces continuous bioacoustic signals. The sound of the blood flow, muscle contraction bursts, and bone crackling are amplified, distorted, and played back through eight loudspeakers using the biophysical instrument Xth Sense, developed by the author. The same bioacoustic data stream excites an OpenGL-generated swarm of virtual entities, lights, and organic forms diffused by a video projector. The work brings together different media so as to creatively explore the processes wherein self-perception, effort, and physicality collide. HC is freely inspired by the sixth Bolgia of Dante's Inferno, located in one of the lowest of the circles of hell. Here, the poet encounters the hypocrites walking along wearing gilded cloaks filled with lead. It was Dante's punishment for the falsity hidden behind their behaviour; a malicious use of reason which he considered unique to human beings" (Marco Donnarumma, "Hypo Chrysos", sito web dell'artista, http://marcodonnarumma.com/works/hypo-chrysos/. Per il video della performance: https://www.google.es/?gfe $\mathrm{rd}=\mathrm{cr} \& e \mathrm{i}=\mathrm{AnDiWPWrAuOs} 8 \mathrm{wed}$ laewCg\&gws rd=ssl\#q=hypo+chrysos\&*.

33. In questo senso, sarà opportuno ricordare che, in ragione della straordinaria e sintomatica inflazione semantica cui si è prestato in anni recenti l'impiego delle parole "performance" e "digitale", l'espressione performance digitale dovrebbe riunire ogni classe di impiego artistico o teatrale di mezzi digitali, ogni tipo di prestazione (artistica, ludica o industriale) afferente alla realtà virtuale, e ogni forma di interattività con il mezzo digitale che comporti un comportamento performativo nell'utente. Per ragioni di spazio, senza dimenticare la rilevanza dello sfondo inflazionario che si è detto, in questa sede mi limiterò a considerare gli aspetti della performance digitale e della cibercultura più vicini alla prassi danzistica. Per l'analisi generale del concetto di arte digitale e per la ricostruzione della sua genealogia nelle avanguardie del XX secolo rimando a Auslander 1999; Grau 2003; Cavallaro 2002; Wesemann 1997. 
it and that volition is expressed through the designated media." 34 Succedaneo di dinamiche celestiali è l'adattamento del rapporto tra performer e intorno digitale a valori crescenti di sensitività e responsività, che a loro volta, con la loro equazione taumaturgica tra obbedienza del sistema e obbedienza al sistema, ricorda beffardamente quell'armonizzazione di spontaneità e disciplina (vera forma strutturale del consenso) invocata da Schechner e da altri a sorreggere l'ossatura ideologica, la dissidenza formale e iper-corporea del nuovo performer: "each move is absolutely spontaneous and part of an endless discipline". 35

Prima di divenire la mascotte Trans e Post-umana dei vangeli cibernetici, il corpo digitale, stick-body o data-body, ottenuto per motion captures, tradotto in immagini computer-generated ${ }^{36}$ o Technogenderbodies, ${ }^{37}$ ha sedotto irresistibilmente le poetiche di fine secolo, che lo leggono come il diagramma ultramondano, terminale e definitivamente formalista di un soggetto emancipato dalle viscosità della materia. La stessa ipotesi di trascendenza elettronica innerva in fondo le tecnologie di simulazione, motion capture e composizione infografica (Life Forms, Poser, Dance Forms, Isadora, EyeCon, Character Studio) $3^{38}$ adottate tra gli altri da Merce Cunningham in Biped (1999). ${ }^{39}$ Lintuizione malinconica che il corpo virtuale possa riassumere più generalmente le ansie suicide di perfezione del corpo post-moderno ha a sua volta ispirato il lavoro di Twyla Tharp: la Golden Section che chiude The Catherine Wheel (1983) è, in tal senso,

34. Estratto da Ellen Bromberg, "Falling to Earth" (1998), Digital Performance Archive: http:// art.ntu.ac.uk/dpa. Un'estensione della stessa poetica (a includere vertiginose modificazioni del percetto corporeo grazie all'intrusione di simulazioni virtuali e proiezioni tridimensionali) si era già dato nel lavoro della Random Dance Company di Wayne McGregor (The Millenarium, 1998).

35. Cfr. Schechner 1977. Schechner non è l'unico dei protagonisti del giro performativo degli anni ' 60 a farsi esponente di un'utopia somatica il cui compimento letterale non sarebbe venuto che con il giro "virtuale" degli anni '9o. L'ascetismo di mezzi invocato in Per un Teatro Povero (1968) da Grotowski (il più antitecnologico dei riformatori) è parte di una metafisica dell'immanenza i cui prolungamenti neo-escatologici calzano a pennello con certe asseverazioni del post-umanismo: "If we strip ourselves and touch an extraordinarily intimate layer, exposing it, the life-mask cracks and fall away" (Grotowski 1968: 19).

36. Esempi di una prassi diretta di ricostruzione interattiva di corpi digitali sono state, negli ultimi anni, le realizzazioni web di Victoria Vesna (Bodies Inc., I995; Notime, 200I) o Nancy Burson (Beauty Composites, 1982), o la nota performance in telepresenza di Orlan (The reincarnation of St Orlan, 1990).

37. La definizione è creata da Kimberly Bartosik (20II).

38. Sarebbe giusto aggiungere a questa lista i CD-Rom e i siti web interattivi che configurano una specie di deriva accademica e pedagogica del concetto di performance digitale, come Improvisation Technologies di William Forsythe (des. Christian Ziegler, 1994), Synchronous Objects for One Flat Thing, reproduced dello stesso Forsythe (Ohio University, 2003) e The Dance Disc di Jacqueline Smith-Autard (Bedford Interactive Institute, 1989). Cfr. SmithAutard 20I4; Forsythe-Haffner 20I2, e il sito: https://synchronousobjects.osu.edu.

39. Intenti analoghi di ottenere un "corpo sottile" si daranno nei lavori sulla motion capture di Bill T. Jones (1999), Yacov Sharir (1997) e altri coreografi della fine del XX secolo. Cfr. Sharir 1997; Kozel 2007: 232-237. 
il più precoce dei "falsi paradisi", tutti oro e alte prestazioni, che nel nuovo millennio orienteranno la lettura dell' ultima Cantica della Commedia..$^{4}$

Se in ultima analisi gli avatar trans-umani e foto-dinamici mobilitati dalle logiche paradossali del "sistema-paradiso" possono leggersi retrospettivamente come la sinopia dei corpi pixelati che danzano nelle simulazioni virtuali degli anni '9o, è perché il trasumanar di Dante negli Oltremondi della teologia medievale, poté a sua volta anticipare, fenomenologicamente, l'olismo di sintesi invocato con insistenza dai cantori della realtà virtuale: "The day when your olographic double will be there in space, eventually moving and talking, you will have realised your dream" (Baudrillard I98I: I05).

Su questa possibilità di piegare le tecnologie alla contraffazione di un simulacro d'anima, "specchiato sembiante" offerto allo spettatore di ciò che sarà nell'insostanzialità dell'altro mondo, si impernia Anima (2002) della compagnia $4 D A r t$, in cui la proiezione $3 \mathrm{D}$ rappresenta suggestivamente la separazione dell'anima degli interpreti (Doppelgänger astrale, scia o traccia grafica) dal loro corpo. ${ }^{41}$

Apoteosi, infine, del transumanismo e paradiso di sintesi sono a modo loro la proliferazione di corpi virtuali del ciberspazio socializzato, ${ }^{42}$ quando

40. Si ricordi, per questo, che nella versione in video la protagonista di The Catherine Wheel, visitando una chiesa "riceve" la visione di uno stick body che rappresenta in qualche modo un modello secolarizzato di "santità" (associato a valori di bellezza, snellezza, prestazione, efficacia, assertività, ecc.). La Golden Section, in cui tutta la compagnia danza freneticamente in un intorno dorato, rappresenta per così dire l'estensione di questa nevrosi paradisiaca a tutto il complesso della famiglia e della società.

4I. Se non sposa direttamente un programma di svecchiamento della vanitas barocca (come accade in Skeletal Reflections di Chico MacMurtrie, 2002; o come, almeno in parte, in Biped di Merce Cunningham), la possibilità di creare in tempo reale un doppio virtuale del soggetto, interprete o spettatore, tende invece a dispiegare un fantasmagorico complesso di trasfigurazioni virtuali (nebulizzazioni, aureole, avatar astrali, animazioni allegoriche o vanitas ecc.); a configurare insomma una forte insistenza ideologica sulla realtà virtuale come "tecnologia di trascendenza", come accade nel lavoro delle compagnie Mesh Performance Partneship (Contours, 1997), Company in Space (Incarnate, 200I), Igloo (Viking Shoppers, 2000), Troika Ranch (The Chemical Wedding of Christian Rosenkreutz, 200I). Una estesa elaborazione teorica delle stesse idee si trova in Kurzweil 2000; Hayles 1999; Dixon 2007: 212-233. Importanti contributi allo studio storico e filosofico del corpo virtuale, o terzo corpo (dopo il corpo fenomenologico e il corpo socialmente costruito), sono anche: Ihde 2002; Mitchell 2003.

42. "Cyberspace [...] involves a curious form of disembodiment, in which each participant becomes a perceptual monad, a concept rather than a physical presence" (Peter Lamborn Wilson, cit. in Negroponte 20I5: 28). Di qui, anche, che paradossalmente solo dallo studio dettagliato dell'arte digitale sia emerso il sospetto che, mentre il Corpo difeso con prepotenza dagli immanentisti di ogni colore tendeva di fatto a comportarsi come una simulazione virtuale, il corpo fatticamente virtuale deteneva una possibilità residua di sollecitare la coscienza dello spettatore sulla pregnanza della sua parte invisibile, del suo rimosso pragmatico, che veniva a essere il corpo fenomenico. E che precisamente nella direzione di uno smantellamento sistematico di questo, che Dixon chiama "mito dell'incorporeità" andrebbe l'attività scientifica e artistica, tra gli altri, di Susan Kozel, Paul Vanouse, Myke Tyler o il collettivo Dumb Type. Su un'interpretazione del mandato della performance digitale volta a ristabilire la pertinenza fenomenologicamente "carnale", la materialità subliminale del 
non il concetto stesso di telepresenza: come argomentai altrove (Fratini 20I4: 99-IOO), rientra nella "fisica dantesca" l'asserzione del dono "beato" di alterare distanze e localizzazioni: il corpo di luce del beato godrebbe del privilegio di essere contemporaneamente nel luogo empirico in cui appare al poeta per interagire con lui nel luogo Empireo che è la suprema corte di Dio; il travaglio scopico di percepire i beati nella distanza è a sua volta totalmente annullato dalla perfetta trasparenza del mezzo attraverso il quale si produce la visione. Difficile non leggere la sottigliezza, immaterialità optico-luminica, interconnettività, funzionalità, automozione e automazione (in ultima analisi, la performance dinamica) del mezzo in cui si sdipana l'ultima parte del viaggio dantesco come l'allegoria avant la lettre del congiunto di qualità che comporranno, nell'ideologia della postmodernità, l'elevata prestazione del medio telematico e dello spazio cibernetico. ${ }^{43}$

Questi i Paradisi - celestiali anche quando sono inferni, e danteschi anche quando non sanno di esserlo - di una parte della danza di fine secolo. Ma per quanti non si lasciano sedurre dall'ipotesi di trascendersi nelle sintesi celestiali della telematica, o che semplicemente desiderano risolvere il paradosso della Commedia di qua dai meccanismi di "alta risoluzione" che, nelle nuove tecnologie, fanno di quel paradosso una nuova coerenza, Dante diventa soprattutto un problema di formato, un dilemma spaziale, un'ordalia del linguaggio (a volte tutte queste cose). Non si potrà immaginarne una versione danzata $o$ performativa senza sostituire alla questione annosa circa il contenuto della sua forma, la questione ben più interessante circa la forma del suo contenuto: la struttura, il continente, il luogo fisico e formale dell'Oltremondo come testo. Non mi riferisco unicamente alle alterazioni della spazialità (altra tendenza dominante negli allestimenti del nuovo millennio), ma al fatto che, se davvero il poema dantesco è, parafrasando Auerbach, una "danza di figure", a venirne perturbata sarà proprio, in prima istanza, la danza come dispositivo figurale $\mathrm{e}$ la danza come topos culturale; di più, se l'Oltremondo dantesco è una sincronizzazione della storia in Giudizio, la Storia che da sincronizzare e giudicare

corpo virtuale, si veda anche Kozel 2007.

43. A sua volta, nel settore di teoria che decostruisce il discorso ufficiale sulle taumaturgie della Rete, è stato possibile additare il contraltare infernale di questa "ottimalită" di prestazione proprio nell'eterna fluttuazione per il cyberspazio, di quelle immagini povere (archivi pirata, immagini a bassa risoluzione, profili falsi, materiale di spam ecc.) la cui tormentosa ridondanza assomiglia tanto a una versione di immortalità appocata da meritare loro la qualifica geniale di "dannati dello schermo". Cfr. Steyerl 20r4. La nozione di "immagine povera" ha a sua volta parentele concettuali con l'idea di "archivio nudo" elaborata da Kenneth Goldsmith. In entrambi i casi, il risultato è la circolazione eterna di questi materiali condannati nei gironi della web, e il vecchio tema della tecnologia come succedanea della religione nell'appagamento di ideali come la libertà, la libertà, la felicità ecc: "es usual que los archivos desnudos pierdan incluso su contexto histórico, se desdibujen y se vuelvan obras que flotan libremente, viajando en círculos a los que normalmente no llegarian si estuvieran vestidos con su ropaje habitual' (Goldsmith 2015: II5). 
nelle versioni danzate del poema, sarà precisamente quella della danza come linguaggio: gioco di scatole cinesi, in cui la danza, figura (sintesi sfuggente, immagine dialettica) dell'umano, persegue nell'Aldilà le figure della figura che è; o va a visitare lo spettrale museo di se stessa.

Non stupisce che come una visita o visitazione si presenti, all'inizio degli anni '9o, anche la più profetica delle versioni danzate della Commedia, la più feconda in tensioni semantiche, e la più rappresentativa della complessità dialettica destinata a retroalimentarsi nei settori "pensanti" della post-modernità, prima che la post-modernità cessasse di pensare e pensarsi.

\section{DANTE: QuASi un'installazione}

Dante in the Elevator. Lindy's Divine Comedy. Ein Stück für I7 Personen und einen Paternoster s'intitola l'esperimento performativo site-specific che l'interprete-coreografa americana Lindy Annis presenta a Berlino nel I991, come secondo capitolo di una Paternoster Trilogy, comprensiva di una Alice in the Elevator (I99I) e di un Columbus in the Elevator (1992) che allude, con ironia teologica, al nome in gergo di un modello d'ascensore comune negli edifici pubblici dei settori occidentale e orientale di Berlino prima dell'unificazione.

Il Paternoster è il tipo di ascensore doppio le cui cabine sfilano in pozzi paralleli, facendosi a vicenda da contrappeso. Poiché ai piani dell'edificio non vi sono porte, ma solo vani aperti che si affacciano ai pozzi, gli utenti prendono al volo l'uno o l'altro ascensore, per scendere o salire. Dante in the Elevator si realizza presso la vecchia sede dell'autorità cittadina per l'Edilizia (Gebäude des Senators für Bau- und Wohnwesen, in Fehrbelliner Platz (ben conservato congiunto urbanistico dell'epoca nazista), scelto perché costituisce, in parole di Annis, una "cattedrale della burocrazia" (Farber 20I5; DepestelVanderbeeken 2015).

L'evento, "viaggio verticale" che si ripete a ciclo continuo nell'arco di una giornata, gioca appunto sulla corrispondenza in un unico movimento tra l'ascensione di una cabina e la discesa (o descensus) dell'altra. All'approfittare della stranezza di una tecnologia in via d'estinzione, irrimediabilmente associata dall'immaginario collettivo allo stile di vita di prima dell'89, Annis sembra pronunciarsi ironicamente sulla storia come falso movimento e sui modelli di circolazione delle ideologie, e ricordare il paradosso che fino a ieri ha concesso, a collettività separate da un muro, di non incontrarsi mai, impegnate, ciascuna per suo conto, a montare sul carro sferragliante dello sviluppo economico o sul perpetuum mobile, sempre reversibile, delle rispettive burocrazie. Di più, l'autorità di Dante offre un referente suggestivo al mito che $\mathrm{i}$ due settori berlinesi coltivarono vicendevolmente, per decenni, in base ai parametri di un dualismo ideologico, che insisteva nel formulare il gap Est-Ovest, 
capitalismo-comunismo (e viceversa) nei termini manichei di un contrasto tra Paradiso e Inferno, tra sviluppo e recessione, tra felicità e dannazione.

La Paternoster Trilogy invita il pubblico a percorrere i differenti piani dell'edificio (sempre un luogo emblematico della topografia politica o memoriale di Berlino), mentre sono i performers a sfilare, a inabissarsi o elevarsi lungo i pozzi degli ascensori. L'esperienza del pubblico è così una captazione parziale, sempre passeggera delle cellule d'azione mostrate dagli interpreti (pose fisse, o azioni semplici e ripetute). ${ }^{44}$ Lo stesso anno, con emblematico tempismo, la brasiliana Regina Miranda presenta al Museo d'Arte Moderno di Rio de Janeiro, una performance-installazione di formato analogo: The Divine Come$d y$ (I99I), pensata per I65 interpreti su 52 postazioni fisse, difende a sua volta l'analogia spaziale tra la tassonomia dell'Oltremondo dantesco e la tradizionale sintassi architettonica dei luoghi di cultura, organizzati per "gironi" tematici e itinerari che corrispondono ai differenti livelli, più o meno visitabili, del museo: così il sotterraneo (la sede "oscena" dei servizi, cioè del lavoro sporco che sostiene il glamour delle esposizioni) ospita le short actions "infernali", i giardini a livello della strada (spazio a cielo aperto, in linea con il poema) trattano del Purgatorio, mentre le sale ai piani alti vertono sul Paradiso. Al pubblico è lasciata una completa libertà d'itineranza. Se Annis intende decostruire in chiave metafisica l'idea di burocrazia, il lavoro di Miranda segna un precedente interessante in materia di associazioni della Commedia alla nozione di museo, catalogo, campionario, repertorio, collezione, Wunderkammer, caravanserraglio, grande magazzino, quando non deposito oggetti smarriti della memoria culturale e della patologia sociale. Pure, che le postazioni degli interpreti siano fisse, o che le loro, affinché nessuno spettatore ne perda i dettagli, siano azioni ripetute regolarmente, preclude all'installazione di Rio gli inquietanti effetti interattivi che dispiega efficacemente, a Berlino, il lavoro di Annis.

Il disorientamento derivato qui dall'impiego del Paternoster, l'impressione di "perdere" costantemente le figure umane appena intravvedute nei frames che danno al pozzo verticale dell'ascensore, anticipa in molti aspetti l'esperienza che, di qui a qualche anno, si avrà al percorrer gli assi orizzontali dispiegati su 19.000 metri quadrati, nel centro di Berlino, dal Denkmal für die ermordeten Juden Europas di Peter Eisenman: la sensazione che sia impossibile seguire fino a destinazione gli sconosciuti (i visitatori stessi del memoriale) che

44. La performance-installazione include anche incontri con ballerini che interpretano personaggi specifici dell'attualità politica e artistica. Barbara Bourget e Jay Hirabayshi, coreografi canadesi, autori delle tre parti di Sunyatak (1989), in cui accade esattamente il contrario: il pubblico è seduto al centro e circondato dai performers. Il lavoro di Miranda e quello del duo Bourget-Hirabayshi sono la dimostrazione di come, a fronte di un immaginario danzistico abbastanza corrivo (soprattutto nelle rappresentazioni del Paradiso, che precisamente per le sue virtù cinematiche è in un certo senso la sfida coreografica più complessa, e quella meno raccolta), si è troppo spesso creduto che la poetica suggerita dall'ultima cantica di Dante potesse essere risolta con questioni di formato. 
attraversano solo di sfuggita gli intervalli tra le 27II steli di cemento del complesso; sensazione di un'immagine vista e perduta nel suo moto di traslazione, di de-portazione verso i punti ciechi dello scacchiere mondiale.

Annis offre così al pubblico la possibilità penosa di strappare all'epifania passeggera dei performers un'immagine enigmatica del senso del presente; l'operazione benjaminiana che è cogliere l'istantanea dei tempi nel momento del pericolo, quando l'immagine della storia è più sfuggente. Di che vacillazione si tratti, e quanto corrisponda alla "fine di un mondo" (secondo alcuni della Storia come l'abbiamo conosciuta), è abbastanza intuitivo nella Berlino del I991. Dante in the Elevator esibisce, per così dire, l'apparizione effimera e ciclica di un Oltremondo che è già il mondo di ieri: quello di due blocchi falsamente evolutivi e fatticamente contrapposti da una sintassi semplice, e semplicemente falsa.

Uno dei gesti-feticcio eseguiti dagli interpreti è quello di chi cerca di liberarsi, con uno scacciamosche, di un insetto che stia aggirandosi nella cabina dell'ascensore. L'azione ricorda il movimento nervoso (un gesto frenetico delle mani all'altezza della nuca, fatto pensando a un insetto) che negli stessi anni, al Frankfurt Ballett, William Forsythe impiega come gesto esemplare del suo metodo di composizione, ricco in patterns di divincolazione frenetica, fondato su un paradigma di irritazione o patologia dinamica, nell'interferenza tra corpo organico e figura, tra carne viva e forma coreografica (Forsythe I991: 49-62).

Parte della dialettica che innerva la ricerca forsythiana, la cui nozione di figura è così strutturale da potersi analizzare secondo l'esegesi dantesca di Eric Auerbach (Fratini 20I4; Auerbach 1966: 174-220), vale anche per il progetto poetico di Annis, incentrato sin dagli esordi sulla nozione di progresso lineare (di qui la fascinazione per i livelli e "strati" dell'assiologia dantesca) e sull'iconografia gestuale, che Annis concepisce - lo farà posteriormente Georges Didi-Huberman nell'ambito di un'ampia riflessione sulle Pathosformeln di Aby Warburg (Didi-Huberman 2002) - come sintomi "ritornanti", complessi di civiltà e somatizzazioni della storia. ${ }^{45}$

Il ciclo verticale del Paternoster (ciclo, cioè dromologia di ritorni; verticale, cioè dromologia di affioramenti e inabissamenti) riproduce con straordinaria economia di mezzi questa "immobilità dinamica", questa recidività fossile dei gesti vivi; e realizza simmetricamente quella mobilitazione della fissità - la condanna di tutto ciò che è transitorio a fissare in eterno la sua transitorietà - che

45. L'interesse di Annis si tradurrà, a partire del 2002, nel dittico scientifico-performativo The Body Archive - The Encyclopedia of Tragic Attitudes (prod. Sophiensaele, Berlino), in collaborazione con Xavier Le Roy (per la prima parte, dedicata alle pose tragiche maschili) e con Joséphine Evrard (per la seconda parte, dedicata alle pose femminili), progetto sostenuto, tra gli altri, dalla stessa Aby Warburg Foundation. 
è a conti fatti la ricetta dinamica, la cinesiologia generale della Divina Commedia. Per quanto le poetiche del Tanztheater siano, per le avanguardie berlinesi degli anni '9o, una cosa del passato, l'interesse di Annis per il conflitto tra il presente della vita e l'anacronismo del gesto (culturale e danzato) sembra rimandare irresistibilmente alla dialettica tra esistenza e danza che ha retto la poetica di Pina Bausch. Se Café Müller (1978) può visitare, foss'anche clandestinamente, questa rassegna di debiti danteschi, è proprio perché vi si rappresenta con eloquenza insuperata il lavorio di un ritorno incessante dei "personaggi" al succinto repertorio delle loro immagini ossessive e gesticolazioni affettive. Come se, nell'anticamera di una specie di eternità, fossero condannati a liquidare un patrimonio residuo di gesti e sensazioni misurandone, a furia di ripeterlo, il progressivo deficit di senso; o rivivendone, a furia di cercarlo, la perdita irrimediabile. Il Tanztheater è soprattutto un dispositivo di trasformazione messo a veicolare la fissazione progressiva dei gesti della vita in figure danzate, o viceversa lo scadimento delle forme della danza in gesticolazioni, tic, agitazioni, sintomi esistenziali: catarsi reciproca di danza e vita; o purgatorio di un linguaggio, su cui brilla, proprio nel finale di Café Müller, la strana consolazione di vederne le immagini definitivamente intercambiabili - la parrucca di Nazareth Panadero sulla testa di Pina Bausch - e le forme definitivamente "circolabili" - la ripetizione infinita di un unico ciclo di gesti degli interpreti nel vano-cabina della porta girevole in fondo alla scena.

Che lo si legga nell'ottica esistenziale di Pina Bausch (che pure è un punto di vista sulla danza), o in quella metadiscorsiva di Forsythe (che pure è un punto di vista sull'esistenza), il modesto esperimento di Lindy Annis è un ammirevole concentrato di tutto quanto la danza postmoderna potesse dire, sin qui, sulla sua omologia con l'Oltremondo in generale e con quello di Dante in particolare.

Sarà forse azzardato, alla luce della sua modesta risonanza nei circuiti della danza internazionale, considerarlo il capostipite legittimo delle imprese dantesche della danza contemporanea durante i tre decenni successivi. Eppure è ragionevole considerarlo un precedente - o una profezia - di molti dei moventi poeticamente negoziabili nella sequela straordinariamente densa di letture, adattamenti e "trattamenti" della Commedia che ho scelto come oggetto di studio, perché enuncia con semplicità parte delle "nevrosi di specifico" (in mancanza di un termine più prudente) che saranno trattate nelle prossime pagine, e che distano dall'essere appannaggio esclusivo delle coreografie d'ispirazione dantesca. Solo interpretando le implicazioni dell'esperimento berlinese di Annis come "questioni interne" della danza di fine secolo si intende con che facilità una parte di quella danza si lasci sedurre dal ricorso a Dante come a un exemplum, mezzo o pretesto di totalizzazione escatologica. 
Non solo perché, già all'inizio degli anni '9o, la caduta del muro fomenta un processo ben avviato di globalizzazione e l'auge di un paradigma storico (o post-storico) abbastanza inedito da indurre tutte le arti alla tentazione "immunologica" di ulteriori sintesi generali e "opere-mondo" (adotto espressamente la definizione di Franco Moretti) (Moretti 1994). Ma perché nel ventennio che precede l'accelerazione culturale della Berlino riunificata, quando totalitarismo spettacolare e capitalismo autoritario sono ancora ipotesi filosofiche relativamente minoritarie, la danza occidentale ha già conosciuto i sintomi di un precoce crepuscolo ideologico: il sentore, tout court, nelle avanguardie degli anni '70 (il Tanztheater stesso, il minimalismo, il concettualismo, l'arte d'azione, ecc.) di una possibile "fine della danza", per esplosione o implosione, per inflazione e/o contrazione, cioè per impoverimento sistemico dei parametri formali di eccellenza che ne marcano lo specifico (una danza sempre meno danzata), e diluizione incontrollabile di quello specifico di bassa intensità nel liquido sinoviale che lo separa da specifici circonvicini (una danza sempre meno danza).

Troppo spesso, quando si considera la stupefacente fecondità degli anni '8o in materia di nuove correnti, si tralascia di sottolineare che quella fioritura di congetture poetiche, tecniche e formati è in buona misura il prodotto convulso di un certo sospetto di "terminalità" dal retrogusto apocalittico; sospetto tout court che la danza debba fare i conti con se stessa; riformulare i suoi dilemmi poetici sub specie aeternatis; sistematizzare la sua storia; prepararsi a sopravvivere nel tempo liquido che viene; finanche rivendicare, fuori tempo massimo, una misteriosa leadership in materia di "universalità": eccellenti ragioni, tutte, per appellarsi in termini più o meno pertinenti all'autorità del maggior poema sacro di tutti i tempi. Non credo di esagerare affermando che parte della danza di fine secolo, sedotta e, almeno in parte, atterrita dall'ipotesi poetica di una dissoluzione accelerata della sua Storia, ma anche mossa da una ferma determinazione a sussistere "con altri mezzi" di là da se stessa, cedesse quasi naturalmente alla tentazione di sdipanare, dispiegare, rappresentare, esorcizzare i suoi Aldilà vari ed eventuali. E che Dante fosse là per oggettivare $\mathrm{e}$, in qualche caso, lenire lo stress di questa escatologia "minore".

Esposti in nuce dalla trilogia di Lindy Annis, i fronti di questa crisi - fatta più di negoziazioni condizionali che di rese incondizionate - che coinvolgerà a vario titolo anche il referente dantesco, possono enumerarsi facilmente:

a) La mise en abîme del rapporto tra immagine e movimento, o tra azione e visione (su cui si negozierà il formato, l'economia spaziale delle future Commedie danzate).

b) La saturazione della memoria gestuale e il problema della storia dei linguaggi corporei come accumulazione degli stili e stallo del giudizio estetico 
(su cui si negozierà l'uso "metadiscorsivo" di Dante come mozione d'ordine rispetto ai bilanci generali e all'archiviazione simbolica della danza occidentale).

c) Il rapporto tra costrutti sintattici verticali e un nuovo ordine paratattico dei segni (su cui si negozierà, anche in danza, la desublimazione, spaziale e tematica, dell'idea d'Oltremondo).

d) La crisi del feticismo teoretico che ha alimentato la nozione di corpo per tutto il XX secolo (su cui si negozieranno gli usi della Commedia come referente generale delle sfocature possibili del corpo, come testura, carne, materia, immagine, ombra, fantasma, ecc.).

e) L'atomizzazione e discontinuità del quadro unitario che ordina la percezione dell'opera (su cui si negozierà la possibilità di rappresentare Dante organizzandolo per moduli, "pacchetti", segmenti, stazioni e postazioni).

f) La riformulazione del ruolo pragmatico della danza nella Cultura contemporanea e fuori dalle cornici accademiche (su cui si negozierà il tenore pedagogico o antipedagogico delle letture danzate del poema).

g) Il ruolo attivo assegnato agli spettatori, e la tematizzazione "escatologica" della nozione stessa di pubblico (su cui si negozieranno le opzioni di approccio interattivo, partecipativo o immersivo alla Commedia);

h) La "crisi d'identità", per così dire, dell'opera stessa: un'attenuazione progressiva dei differenziali morali, metafisici, dinamici ed estetici che ne organizzano la sintassi (su cui si negozieranno la possibilità di ribaltare l'interpretazione tradizionale dell'Inferno o del Paradiso e quella, più in generale, di disordinare la materia del poema in un tutt'uno entropico).

La prossima tappa del nostro viaggio passerà per alcuni di questi teatri di guerra.

\section{DANTE: QUASI UNA META-COREOGRAFIA}

Di che pervicaci turbolenze si compone, a grandi linee, il clima artistico d'inizio millennio? Estenuazione del buzz millenarista, saturazione delle forme, inflazione dei segni, ammutinamento dei parametri, stagnazione delle poetiche, miopia dialettica, stress dei linguaggi: è paradossale che in uno stadio così pelvico e definitivo della Storia d'Occidente, segnato dalla popolarizzazione a marce forzate del ballo e dei suoi usi (commerciali, simbolici, sportivi, mediatici, terapeutici), la teoria saluti con tenerezza gesuitica il disbrigo concettuale dell'"esaurimento della danza" inaugurato dalle avanguardie degli anni '9o e perpetuato da allora con zelo impiegatizio in tutte le succursali accademiche e pratiche della decostruzione. L'entusiasmo diffuso per l'ascesi poetica - di fatto l'anemia - che sdilinquisce una parte considerevole dell'avanguardia, è perfettamente funzionale all'ascesa di una danza popolare che letteralmente, nella prassi e nei consumi, scoppia di salute. La danza d'arte vive una stagione 
di rigori purgatoriali e radicalismi programmati, deprimente per tutti fuorché per le anime belle ancora persuase, in arte come in politica, del potenziale di redenzione dell'austerità.

E mentre nei cenobi della sperimentazione si ama credere che la povertà generalizzata dell'offerta artistica sia una tuonante dichiarazione di dissidenza contro l'assolutismo televisivo di una volgarità che ha scelto proprio la danza come veicolo di contagio, artisti di spiccata intelligenza, all'inizio del millennio, intuiscono che la gravità del momento dipende meno dall'insidia di forze esterne che premono per vanificare l'avventura spirituale di cent'anni di danza moderna, che dalla forte tendenza interna di quella danza a compiacersi della paralisi, a tradurre il disorientamento in cortocircuiti del senso e atrofie dell'azione, a coltivare intorni di creazione sempre più arbitrari, a sdipanare il suo dopostoria in un catalogo inesauribile di dotte minuzie.

Persa in una selva inflazionaria di segni, di memorie immediate, di stimoli a corto termine, la danza d'Occidente ricorda tutto sommato il Dante del Canto I. Paralizzata da una tentazione ipertrofica di movimento, ne impediscono la velleità di progresso il sovraccarico della memoria propria, e l'oscena realizzazione delle sue utopie di democratizzazione nel trionfo indiscriminato delle danze commerciali di ogni tipo.

Selva selvaggia e aspra e forte. Quasi più amara di quella morte che la danza aveva paventato nelle ore corrusche del suo crepuscolo, fra gli anni '8o e i '9o, quando il sentimento della fine suggeriva ancora, ad alcuni, le più geniali involuzioni poetiche.

I tempi sembrano maturi per operazioni confessionali di riordinamento (forse anche di catarsi o decongestione) nelle forme doverose dell'osservatorio ultramondano: di un descensus della danza al paradigma saturato delle sue forme, che implicherebbe di smascherare concettualmente l'ossessione evolutiva che ha definito la sua vitalità in occidente; di ripensare la postmodernità come la forma ultramondana, la rivelazione del suo eterno ritorno su se stessa, come la retribuzione tormentosa degli abbagli somatici in cui si è compiaciuta; $\mathrm{e}$ in fondo come la realizzazione infernale dei suoi desideri più selvaggi e delle sue migliori intenzioni. Fare i conti con se stessa, forse. Ma soprattutto farsi debitrice a Dante della maggiore acrobazia mentale: andare all'altro mondo pur di sopravvivere, e sopravvivere pur di andare all'altro mondo.

Trasformando Dante in un'arma radicalmente metadiscorsiva per dire che l'unico inferno danzato è proprio la "danza come inferno", la trilogia sulla Commedia di Emio Greco e Pieter C. Scholten segna, tra il 2008 e il 20II, un vero turning point nella storia degli allestimenti danteschi. È altresì l'allegoria calcolata del passaggio della compagnia a una seconda maturità, segnata dall'irraggiarsi sistematico delle sue poetiche su differenti domini segnici (opera, composizione musicale, cinema, poesia, filosofia, fotografia, peda- 
gogia), e dall'intuizione dell'urgenza di una super-visione del mandato della danza nella cartografia dei récits che possono concorrere alla rappresentazione del mondo. Cuando Scholten dichiara che "La danse est certes un art, mais aussi un savoir", ${ }^{6}$ chiama in causa l'allure totalizzante di un'avventura coreografica che condivide con il referente dantesco la sottile arroganza di essere allo stesso tempo Opera e Mondo, Poesia e Summa, congiunto sistematico di esperienza e saggezza, di sviste carnali e di saperi somatici: "Langage du corps recréé à travers tous ses possibles, toutes ses cultures". L'Oltremondo è destinazione finale e organizzata di tutti i regimi del corpo, di tutti i suoi stili e paradigmi, erranze ed errori.

È come se le memorie cinetiche d'Occidente avessero sviluppato una specie di saturazione termodinamica: tutto quanto poteva fino a pochi decenni orsono cristallizzarsi in paradigmi specifici (scuole, stili, correnti, generi) ha raggiunto e superato, se si vuole, il suo "punto di fusione". Il successo indiavolato, nella prassi recente, dei cosiddetti esperimenti di fusione, non è che uno dei sintomi falsamente incoraggianti di questo surriscaldamento delle poetiche che, al liquidare il prestigio della sintassi, liqua anche tutti i parametri di coordinazione, subordinazione a trasmissione che l'hanno accompagnata.

Non può stupire che in Hell (2006) ${ }^{47}$ di Greco/Scholten la scenografia si riduca quasi esclusivamente a un albero spoglio: espediente polisemico, senza dubbio (il peccato originale, la selva oscura di Inferno I), ma anche sineddoche della foresta di stili in cui si inscena la parabola di quest'inferno danzato. L'albero di Hell finisce per incarnare una maledetta nostalgia di ramificazioni perdute, di genealogie smarrite, e di quei valori di procedenza e discendenza che hanno permesso in passato, alla danza occidentale, di pensarsi (o di favoleggiarsi) come la Storia di qualcosa. E se la selva dei suicidi del dettato dantesco può assumersi tra i referenti della sua desolazione, è perché Greco non sembra essere insensibile alla fascinazione di un apologo, quello di Inferno XIII, che descrive la pena come uno stato irreversibile di spossessione di sé, di auto-alienazione del corpo nella forma, "a jungle of dark essences". ${ }^{8}$ " D'abord la perte de la ligne droite" recita Greco, parafrasando a modo suo il terzo verso

46. Sullo stesso tema in merito a [purgatorio] Popopera, Greco 2008: "Cet apprentissage musical et cette confrontation du corps avec l'instrument, c'est également une manière d'être fidèle à Dante, dans une part important de sa quête: l'idée que le voyage entraîne une forme de connaissance qui passe par la maîtrise des arts, de la science, et constitue donc un savoir."

47. Prod. Cia EG | PC, Théâtre de la Ville, Festival Montpellier Danse, Maison de la Culture Amiens, barbicanbite07, Cankarjev Dom, Julidans 2006, creaz. Festival Montpellier Danse. Hell sorge da un esperimento in forma di solo come Double Points: Hell (2005) creato per le Sujet à Vif al Festival d'Avignon. L'informazione sullo spettacolo fornita dal video d'archivio della compagnia (e dalla mia personale visione dello spettacolo al Théâtre de la Ville nel 2007) è stata completata dal documentario Imagined Hell (Prod. Las Negras, 2008) di Maite Bermúdez, sul processo di creazione di Hell: https://vimeo.com/37046966.

48. Testo del programma dello spettacolo, Emio Greco | PC, 2006. 
del poema ("Che la diritta via era smarrita"), in uno dei titoli apposti all'antologia di fotografie della fanzine dedicata dalla compagnia al progetto Hell. ${ }^{49}$ La lettura dell'Inferno di Greco e Scholten riscrive il concetto auerbachiano di Figura nella frizione tra necessità organiche e slanci dello spirito ("une allégorie de la lutte individuelle et discrète contre la dispersion de la chair toutes causes confondues [...] lutte contre le corps indexé"; Goumarre 2006: 34), tra le strutture di ritorno dell'ereditarietà - biologica o stilistica -, e la linea di fuga anti-biologica del desiderio, del fantasma, dello stile individuale.

Hell rappresenterà dunque meno un insieme ridotto di "corpi all'inferno" che un repertorio eccezionalmente vario di "inferni nel corpo". L'Oltremondo sarà dunque un atto di somatizzazione: comportamento forzoso di corpi sequestrati da un paradigma gestuale o energetico, che è allo stesso tempo l'allegoria dinamica di una "norma di castigo" e l'allusione diretta a un certo tipo di stile storico, di "comportamento danzistico": "vizio di forma", "vizio $d i$ una forma", finanche circolo "vizioso" dei singoli stili come ritorni, ricorrenze di una perversione inscritta, cartografata nel corpo.

Il descensus di Hell esibisce, almeno nelle intenzioni e ispirazioni, un'analogia inedita tra quei circoli viziosi (che determinano i generi sovrapersonali e le tendenze capitali della danza d'Occidente) e l'ordine infallibile dell'eredità genetica (anch'esso una spirale, un descensus di aminoacidi) in cui si articola l'inferno del corpo di ognuno, la cattura della sua storia nella sovradeterminazione degli statuti biologici: "L'histoire familiale et individuelle se pare d'une histoire génétique, rendue objective par le décryptage des gènes et l'explication chimique de nos forces et de nos faiblesses" (Néri 2006: 22). Quasi negli stessi anni Romeo Castellucci ha dedicato a paradossi bio-tragici le sequenze di aminoacidi e la disperazione genomica di Tragedia Endogonidia (2002-2004); e un'allusione alla manipolazione plastica, genetica o protesica del corpo come inferno (Capaldi 2006: I89-I9I) è nel centro della coreografia di vaga ispirazione dantesca, quasi contemporanea di quella di Emio Greco, Nel foco che gli affina - Reinigende Feuer (2006) del canadese André Gringas. ${ }^{\circ}$ Dell'idea dell'inferno come protocollo individuale di somatizzazione di patroni o dia-

49. "Il est question de chemin, de forêt, actualisé par la perte d'une 'voie droite', de telle sorte que ce paysage figuratif tracé rapidement par Dante apparaît comme le résultat de la disparition du trait de l'abstraction: la droite... une déroute en somme, une droite qui irait se perdre, se tordre dans le dispositif infernal de 'cercles', lignes sinueuses et autres circonvolutions" (Goumarre 2006: 34).

50. Prod. Korzo Productions, Stadttheater Nürnberg; creaz. Stadttheater Nürnberg, I4 gennaio 2006. Attivo, in questo come in altri lavori, sul fronte della critica agli eccessi della biotecnologia e dell'ingegneria genetica, Gringas (a sua volta ex interprete di Bob Wilson) appare deciso a rafforzare l'analogia tra inferno e consumo; meglio, a sottolineare l'analogia tra la consunzione letterale, lo sfacelo dei corpi nelle parti basse dell'Oltremondo dantesco e l'ordalia del corpo della civiltà capitalista avanzata, "lavorato" incessantemente dal consumo di sé nelle forme della chirurgia plastica, della modificazione genetica e del perfezionamento protesico. Cfr. Heinold 2006. 
grammi generali danno ragione, Greco e Scholten, nella costruzione della danza dei singoli interpreti, esortati a dedurre dalla situazione dinamica dei dannati di differenti gironi della prima Cantica un modo caratteristico e peculiare di presenza e movimento, che tenderà a sua volta a evocare distortamente il modo "generale" di movimento e presenza inerenti a questo o a quello stile riconoscibile di ballo (così, per esempio, la pena di Pier della Vigna suggerirà a un interprete movimenti di frammentazione segmentale o di contorsione che rimandano a loro volta alla memoria cinetica del flamenco)..$^{5 \mathrm{I}}$ Appassionati di neologismi dialettici, Greco e Scholten chiamano extrêmalism (una summa di estremismo e minimalismo) questa facoltà, nell' inteprete, di epokhè gestuale: di estrazione, depurazione e amplificazione del diagramma dinamico che tradisce un pensiero, uno stato psichico o un significato.

Allo spazio di escursione e intervallo dialettico che si dà fra il travaglio di somatizzazione individuale e gli effetti di somatizzazione generalissima e menzognera degli stili di danza storici e commerciali - all'escursione, se si vuole, tra individuazione e appartenenza -, è dedicato il memorabile introitus di Hell, che accompagna la lunga entrata del pubblico in sala con la spossante rivista in cui i danzatori offrono, a scopo d'intrattenimento, una chiassosa suite danzata su grandi successi $p o p$, una vertiginosa rassegna di stili di danza commerciale (dal swing, al soul, al funk, al flamenco, etc.), tutti incarnati con impeccabile esibizionismo. Quando la kermesse euforica di questo prologo si esaurisce e si spengono le luci di sala, la sensazione è che tutta l'energia degli interpreti sia già stata spesa, e che Hell aggiungerà solo ulteriori finezze e perversioni, giri di vite e gironi di vizio a questa tortura dei linguaggi (la tecnica classica, uno degli stili di formazione di Greco, vi svolgerà un ruolo squisitamente tormentoso, come quando tutti gli interpreti si sfiniranno in una interminabile batteria di entrechats).

Per quanto possa apparire peregrino, il prologo spettacolarmente indiavolato di Greco e Scholten capta un sottinteso esegetico della rappresentazione culturale dell'Oltremondo cristiano (e per estensione di quello di Dante), che è passato paradossalmente inosservato alla maggior parte dei creatori visti sin qui: mi riferisco alla dottrina della "performatività" infernale. Non mi riferisco tanto alla "spettacolarità" metaforica dell'Inferno dantesco (gettonatissimo incentivo di mille adattamenti), quanto alla sua spettacolarità letterale: la possibilità di immaginarne le pene come una forma di intrattenimento, uno spettacolo crudele, la prestazione di una moltitudine di corpi condannati alla spietata purezza di una pura performance in cui lo spettacolo della pena sia

5I. Materiali dello stesso tipo sono, in Hell, i movimenti sinuosi dettati dalla descrizione delle metamorfosi in serpenti di Inferno XXIV-XXV, o il vocabolario di mutilazioni simboliche di Inferno XXVIII. 
anche la pena reale di un corpo. ${ }^{52}$ L'installazione di lampadine, destinata a essere leit-motif plastico della trilogia, ${ }^{53}$ che domina il lato destro della scena e che costituisce l'unico accesso all'Inferno metadiscorsivo di Greco/Scholten, è allo stesso tempo porta del palcoscenico di un teatro di varietà, specchio di camerino e sineddoche sarcastica della sostanza luminica, elettrica, taumaturgica di un dio sempre più secolarizzato nei miracoli della tecnologia e sempre più identificato con l'eminenza concertante, il regista di questo show del parossismo. Se dunque la porta di Inferno III dichiara il suo "alto fattore", è teo-logico che qui il regista dello spettacolo, Pieter Scholten (inaugurando una serie di cammei e interventi fugaci che sarà la costante della trilogia),54 quando una lampadina difettosa dell'installazione inizia a sfarfallare, entri in scena dalla platea, come un dio desublimato, a riavvitarla.

Se a tratti lo stesso Dante (in episodi come l'incidente tra Dante e Filippo Argenti nella palude Stigia, o le acrobazie slapstick della bolgia dei barattieri) ${ }^{55}$ poté esibire il clima di spietato siparietto ultramondano che Greco e Scholten colgono così bene, è perché la Scolastica medievale lo aveva a sua volta già introdotto come argomento teologico. All'indignazione - nel De spectaculis e in altre opere di Tertulliano $-{ }^{56}$ per il ludibrio cui si esponevano i primi martiri, dati in pasto al gusto pornografico delle masse per lo splendore dei supplizi nelle arene romane, risponde, nella Summa teologica di Tommaso d'Aquino una tetra profezia di retribuzione: la certezza che il tormento futuro dei nemici di Cristo, tutti destinati all'Inferno, potesse essere uno spettacolo abbastanza balsamico da annetterlo senz'altro al catalogo di squisitezze paradisiache promesso a quanti oggi soffrivano in circhi e anfiteatri. Il loro godimento più raffinato sarebbe stato poter osservare dall'alto delle tribune del Paradiso lo strazio degli infedeli nell'abisso. ${ }^{57}$ Nell'ottica di Greco e Scholten quest'ordine di inversione e retribuzione si vede arricchito da un ulteriore livello di complessità, che rimanda al protocollo specifico dell'interferenza tra lo stile caratteristico di Emio Greco (un postclassicismo assai raffinato, fatto di minuzia e

52. In questo, Greco ripropone con mezzi originali l'impressione che la danza potesse a sua volta immaginarsi come il centro di un dispositivo "infernale" di spettacolarizzazione dello stress da ballo già suscitata da They Shot Horses, Don't They? (1969), il memorabile film di Sidney Pollack sulle maratone di danza statunitensi della Gran Depressione. Un'analisi dettagliato del fenomeno si trova in Bertin-Bertin 2014.

53. La stessa installazione apparirà velata, come un'accenno di costellazioni, nello spettacolo dedicato alla seconda cantica; e sarà la protagonista del finale di YouPARA/DISO, ultimo episodio della trilogia dantesca (di fatto una "tetralogia", se si considera que Greco tratta il Purgatorio in due fasi).

54. In [purgatorio] In Visione Scholten apparirà seduto sulla sommità di una scala, e in un momento parrà dirigere l'orchestra da camera che accompagna l'assolo di Emio Greco. La sua ombra passerà per l'ultima volta dietro le cortine che circoscrivono lo spazio di YouPARA/DISO.

55. Analizzo questi passaggi di Inferno VIII e XXII in Fratini 20I4: 9I-93.

56. Soprattutto Ad Martires, Apologeticus e Ad Nationes, tutti apparsi nel 197 a.C.

57. Cfr. Summa Theologica, Suppl., q. 94, a.I, cit. in Agamben 2007: I8I. 
frenesia, cui calza a pennello l'etichetta di "estremalismo" che si è detta) e la centuria degli stili commerciali da cui si lascia contaminare: se durante tutta la sequenza d'inizio lo stesso Emio Greco appare come un'ombra a doppiare i movimenti della danza di alcuni degli interpreti, nel corso dello spettacolo potrà accadere che alcuni dei movimenti o delle qualità riconoscibili come parte essenziale dello stile di Greco, vengano doppiati, distorti, pervertiti in direzione mimetica dal corpo degli interpreti. Come se il destino dei corpi fosse di essudare in ogni istante la quintessenza ombratile, il fantasma della forma che li esaurisce (il paradigma del loro rigore), e viceversa, ogni forma fosse destinata a "cadere" nella concrezione somatica che la rende riconoscibile, rutinaria, ripetibile.

La danza (un tema che fu già caro a Pina Bausch) è il doppio spettrale ed escatologico, il Doppelgänger della vita. Così, lo iato in cui poteva compiacersi Tertulliano tra corpi gloriosi (che solo osservano) e colpi dolorosi (che solo soffrono) si irradia di questa ambiguità ulteriore, che include nella dannazione di danzare la condanna a godere un po' abiettamente, da attori e spettatori, di questa esazione interminabile che la forma esegue sulla carne, quando un'ombra "cattura" il movimento dei singoli corpi, o quando viceversa una specie di smania mimetica fa modellare il comportamento di ciascuno a imitazione di quello di ciascun altro.

Il risultato sono alcuni degli unisoni "imperfetti" più brillanti della danza recente: vera e propria decostruzione antieroica del sinfonismo orchestico, da una parte, e delle presunzioni del solismo dall'altra. ${ }^{58}$ All'Inferno (Dante lo sapeva bene) è impossibile, da un punto di vista dottrinale, stabilire se la sostanza spettacolare della pena individuale risieda nell'immagine di un corpo o nel "corpo di un'immagine"; che il tormento vi sia indiscriminato, spersonalizzante e comune è parte, appunto, di questa rimozione, essenzialmente performativa, del discrimine tra corpo e spettacolo, tra prestazione e prostrazione. Solo il corpo che non ha già nulla da perdere, neppure se stesso, può perdersi così nella precessione delle sue immagini, delle sue figure danzate.

Lidea di Transpaso (sic), intorno a cui gravita la poetica generale di Emio Greco - versione somatica, se si vuole, del trasumanar di Dante, afferisce a questo processo palindromo di "decantamento": dei materiali dell'esistenza sussunti in forme cinetiche (che ne saranno la flagranza) e delle forme cinetiche distillate a loro volta in tracce organiche ed esistenziali (che ne saranno

58. Non è un caso che la musica di Hell, elaborata da Stephen Greco, si articoli attorno alla modulazione, alterazione, deformazione e in qualche caso "esasperazione" della Sinfonia Eroica di Beethoven, il cui ruolo simbolico nella dinamica del pensiero d'Occidente attorno agli eroismi e alle abiezioni del concetto di individualità è noto. Si può pensare alla nozione, forgiata da Donald Hutera in riferimento al lavoro di Emio Greco, di "explosure" (contrazione di "esplozione" e "esposizione"). 
la fragranza). ${ }^{59}$ Nell'antropologia di Greco/Scholten la danza, organizzata così spesso attorno alla sfuggevolezza, verrebbe a occupare questa posizione di medialità: un'ombra, un "sentore" altamente instabile, minaccia persistente di condensazioni e volatilizzazioni o, in ultima analisi, di inferni e paradisi. ${ }^{60}$

Aver attinto con acume agli statuti della danza commerciale il vincolo reversibile di danza e consumo (che fa tutt'uno della danza che si consuma e di quella che consuma), permette anche a Greco e Scholten di affrontare con un certo slancio l'imbarazzo che ha afflitto sin qui la maggior parte dei lavori di scenificazione della Commedia: quello di dare ragione della materia della seconda Cantica. E benché non evitino la tendenza generale a contrarre il margine di autonomia poetica del Purgatorio rimandandone lo statuto di transitorietà e precarietà a una dipendenza generale dalle due cantiche esterne, ${ }^{6 \mathrm{I}} \mathrm{è}$ un fatto che il loro Purgatorio doppio è a tutt'oggi il più esteso degli omaggi coreografici alla seconda Cantica e al suo clima psico-poetico, fatto di pazienza e di temporaggiamento.

Se dal baccanale di dissipazioni di Hell e dalla sua cartografia dialettica si è potuto dedurre un additamento degli aspetti infernali della danza nella tensione semantica tra ozio e vizio, tra libertà e coazione, è logico che la poetica di [purgatorio]POPOPERA (2007) ${ }^{62}$ e del successivo [purgatorio] IN VISIONE (2008) verta, perpetuando un altro aspetto della nozione di transpaso, sull'idea della danza come lavoro, travaglio, disciplina, penitenza; e sulla sensazione

59. Non deve stupire, pertanto, che nel contesto degli atti di comunicazione e promozione che circondano il progetto della trilogia, e in ossequio a un certa volontà di esplorare le possibili risonanze del lavoro coreografico in domini segnici inusuali, Greco e Scholten decidano di lanciare commercialmente un profumo (Extrêmalism) elaborato dall'artista Maki Ueda sulla traccia olfattiva del sudore degli interpreti, a partire dai costumi dello spettacolo. Dei costumi usati nelle creazioni dell'ultimo decennio, e della loro traccia olfattiva si compone anche l'installazione Double Points: remains (2003): "Usure, labeur, sueur léger mur d'étoffe formé par les tuniques des créations passées" (Filiberti 2006: 27). Cfr. Vigier 2008. La poetica di Greco e Scholten (articolata nel ventaglio di attività dell'ICKamsterdam, e soprattutto nel progetto di neo-notazione Capturing Intention, frutto della collaborazione con l'assistente Bertha Bermúdez) punta risolutamente a creare superfici d'iscrizione non solo per il movimento fisico e fattico, ma anche per il pensiero soggiaccente all'atto di formalizzazioneincarnazione che è la danza: "Inside Movement Knowledge", oggetto del Notation Research Project o Capturing Intention. È parte del progetto un lavoro sulla distorsione dell'immagine propriocettiva inerente a alcune malattie psichiche.

6o. Un'ambivalenza analoga è parte dell'apprendistato di Greco come interprete-coreografo (nella cui pratica giovanile entrano non pochi generi "minori", come il cabaret), formato in poetiche divergenti e diversamente estreme, come quella "iper-corporea" di Jan Fabre da una parte, e quella di Saburo Teshigawara ("ipo-corporea", se si vuole, incentrata sull'inevidenza ottica del corpo scenico), abituato a transitare un certo dualismo, tra tecnologia e sangue, tra virtualità e carnalità 0 , di nuovo, tra Paradiso e Inferno.

6I. "[purgatorio] POPOPERA, qui est encore un peu l'Enfer, et [purgatorio] IN VISIONE, qui est déjà un peu le Paradis", "Entretien avec Emio Greco et Pieter C. Scholten", dossier Festival d'Avignon, 2008.

62. [purgatorio] POPOPERA, Prod. ICK, Holland Festival, Théâtre de la Ville, Festival d'Avignon, Maison de la Cuture d'Amiens, Torino Danza, creaz. I9 giugno 2008, Amsterdam. 
che questa economia cinetica del Purgatorio, emblema di tutte le alleanze tra danza ed etica lavorativa, rimandi in prima istanza al diritto-dovere di spezzare l'ordine di viziosità dei circoli e gironi sanciti dall' Inferno: "whereas in HELL we let our dancers wander round the same circles each time, in [purgatorio] POPOPERA they break out of them. The will, the need to live and especially the hope for the future are the essential motives" (Scholten 2008).

Greco non sembra ignorare che la vera mutazione genetica della danza, nel passaggio dalla classicità alla modernità, fosse da attribuirsi a un inedito entusiasmo per il concetto di sforzo (il "diagramma di sforzo" labaniano ne sarebbe stato, già negli anni '20, la trascrizione concettuale) (Laban 1994; Maletič 1987: 99-IOI; McCAW 20II) e per tutto quanto potesse agglutinarsi a questa area semantica: il prestigio delle cause (che spazzava via i presunti effettismi puri del balletto); la sincerità della prestazione fisica (che spazzava via le presunte frivolezze aristocratiche della danse d'école); il culto puritano per la creazione metodica e cumulativa (che riammetteva la danza alla dignità del lavoro); la riabilitazione dello spazio naturale (che negli assoli di Duncan spazzava via le luci presuntamente bugiarde dei teatri ottocenteschi); la riabilitazione della danza stessa e dei suoi rigori schietti come forma di rieducazione, purificazione, correzione, redenzione o cura (che superava le degradazioni organiche della danza sulle punte); i mille miti di sobrietà produttiva e trasparenza delle intenzioni (che scrostavano il presunto decorativismo malintenzionato degli stili classici); la difesa dell'equazione involontariamente monastica tra forma dell'azione e forma della vita (forma vitae) tout court (che invalidava la dissociazione tra corpo quotidiano e corpo ballerino); la virtù come antidoto al virtuosismo; finanche il sospetto (destinato a godere di un prestigio crescente) che la sostanza della danza più sincera dovesse ricercarsi fuori dalla danza "danzata", nelle pedestre verità di azioni semplici, tasks elementari e docili ripetitività. Aspetti di un'ideologia generale della danza moderna che venivano a costituirla quasi per intero come una dimensione "purgatoriale" nei termini eminentemente stabiliti dalla seconda cantica dantesca. ${ }^{63}$

Istanze di dignità lavorativa tornano anche nelle glosse di Emio Greco alla poetica di POPOPERA: “J'ai toujours été sensible au fait que le danseur était aussi un travailleur. La classe ouvrière est là, en lui, à travers son endurance, l'acharnément de la répétition du travail. Je crois qu'on atteint là une expérience à la fois philosophique et physique. En travaillant ainsi physiquement, le danseur renouvelle sa pensée. Il ne s'agit pas tant de virtuosité, ni de force pure, que d'un moyen d'atteindre une connaissance plus critique. Un corps qui danse devient plus intelligent" (Greco 2008).

63. Sulle analogie tra paradigmi cinetici del Purgatorio e poetiche del movimento nella modernità, cfr. Fratini 2014: 95-96; sulla genealogia di una coscienza lavorativa della danza moderna, cfr. Franko 2002 e Pouillade 2009. 
Ma poiché questa intelligenza del corpo passa per affinamenti, adattamenti, "accordature" progressive, analoghe a quell'intelligenza di Dio cui preparano i lavori forzati della montagna del Purgatorio, fino all'ultima estenuazione dei penitenti "nel foco che gli affina" ( $P g$. XXVI, I48); e in ossequio alla lettura tradizionale del Purgatorio, fatto di concentrazione e concertazione (con i suoi canti, inni, cori e preghiere collettive) come una dimensione soprattutto corale, musicale e concertante, [purgatorio] Popopera sposa l'idea di sottomettere il corpo dei ballerini alla disciplina oggettiva di uno strumento esterno al corpo, e di fare loro interpretare dal vivo la musica per chitarre elettriche composta da Michael Gordon, in un tour de force che obbliga ciascuno non solo a apprendere tecniche basiche di esecuzione musicale, ma anche a integrare gli strumenti al movimento. ${ }^{64}$

Vi è, nell'armonizzazione faticosa e tenace del danzare e del suonare, qualcosa del dualismo che la regola benedettina espresse nel celeberrimo "Ora et labora"; qualcosa, più in generale, del paradosso che configurava lo stile d'esistenza degli ordini monastici come la scelta di una forma vitae: terreno di fusione definitiva, e in molti aspetti performativa, tra le esigenze oggettive della forma (la regola stessa) e quelle soggettive della vita individuale (Agamben 20II); la faticosa interiorizzazione di una disciplina severa che poteva dirsi compiuta solo nel momento in cui la strumentalità della regola appariva come un'estensione naturale della volontà del monaco, un poco come, in POPO$P E R A$, il lavoro di mortificazione può dirsi compiuto solo nel momento in cui la "protesi" dello strumento musicale appare come un'estensione organica del corpo degli interpreti. Greco ha probabilmente in mente questo itinerario di "ammortizzazione" dell'obbedienza in fervore, e del lavoro in orazione, quando si riferisce al gruppo degli interpreti come a una "fratrie un peu tribale et primitive". E ad esprimere una tensione penitenziale che era in fondo il sottinteso ironico delle maggiori creazioni anteriori (Rimasto Orfano del 2002 e Congiunto di nero del 2004) Greco prescrive anche a POPOPERA il tipo di costume unisex - quasi un uniforme - già brevettato in quegli spettacoli: tenuta che nella foggia, nel taglio, nella testura e nel colore ricorda un saio monastico tagliato alle ginocchia, paludamento penitenziale e abito da lavoro allo stesso tempo.

In un ruolo che può far pensare alla Matelda dantesca, l'artista d'opera Michaela Riener passeggia cantando per questa tempesta di suoni elettrici quasi a rappresentare l'altro polo di una dialettica ponderale (tra la pesantezza del sound rock e la leggerezza del canto lirico, tra i rigori della penitenza e le consolazioni del paradiso terrestre), in una funzione che è il correlativo teatrale

64. Quando Greco e Scholten elaborano la loro versione della seconda Cantica, è ancora fresca la memoria della triade di doppi albums dedicati dal gruppo tedesco di musica elettronica Tangerine Dream alla materia dantesca: Inferno (2002), Purgatorio (2004), Paradiso (2006). 
della funzione visiva svolta, qui, dal grappolo di globi bianchi che sovrasta la scenografia.

Chitarre e globi appariranno inerti e stesi al suolo, nello spazio di [purgatorio] IN VISIONE (2008), ${ }^{65}$ ulteriore spettacolo dedicato alla seconda Cantica, stavolta nella forma di un recital solistico dello stesso Emio Greco. Ha senso supporre che Greco intendesse completare l'esperienza di POPOPERA con una versione intimista dello stesso universo penitenziale: che si trattasse, dopo l'uso protesico e penitenziale del fardello delle chitarre nello spettacolo precedente, di insistere sulla rinuncia a ogni proprietà terrena (compresa quella dell'eccellenza scenica) e dipingere l'ascesa alla montagna del Purgatorio come un itinerarium mentis in deo fatto di distacco progressivo dalle passioni ${ }^{66}$ e di un'amplificazione contestuale degli aspetti cosmici e sferologici dell'Oltremondo. Di qui che In VISIONE accentui la sproporzione tra le dimensioni coreografiche dell'evento (un solo contemplativo) e i suoi referenti estetici immediati (come la Matthäus Passion di Bach nell'adattamento di Frank Krawczyk per l'Amsterdam Sinfonietta); tra la piccolezza e relatività della figura umana (ne fanno fede le allusioni al Charlot chapliniano e gli altri elementi clowneschi della performance di Greco) e l'incommensurabile perfezione delle costellazioni i cui moti, affidati a solenni giri perifrastici, circoscrivono tutta la temporalità del Purgatorio dantesco; tra la solitudine dell'interprete-coreografo, lasciato solo a fare i conti con gli strumenti della sua danza, e il sussurro della realtà, immane testura di sussurri e voci radiofoniche o televisive in cui si impasta la musica dello spettacolo. Tutto è fatto, in questo lungo esercizio di meditazione, per mantenere intatto un certo sentimento di sospensione didattica, di relativizzazione e procrastinazione (la stessa musica di Bach è posta come in loop, come se se ne volesse ridurre al minimo il potenziale di narratività).

Due anni dopo, Greco e Scholten chiudono l'esperimento dantesco con youPara/Diso (2010), ${ }^{67}$ dedicato alla terza Cantica. Coscienti di avere conseguito la migliore approssimazione possibile a una certa contemplatività nel loro

65. Prod. ICK, Holland Festival, Thëâtre de la Ville, Automne en Normandie, Maison de la Culture d'Amiens; creaz. 13 giugno 2008. L'assolo di Emio Greco sulla Matthäus Passion di Bach è stato recentemente alla base della creazione per sette interpreti Passione (2015), prod. Ballet National de Marseille, creaz. Opéra de Marseille, 28 novembre 2015.

66. "The pain of the post-industrial human is phantom pain, for he suffers from something that is not his and that never really will be. He buys his emotions, which is why he also clings to them so tightly. These days a person who says: 'I have a pain', is quite literally saying: 'I am the owner of this or that pain"' (Grunberg 2008).

67. Copr. Monaco Dance Forum, torinodanza, Maison de la Culture d'Amiens; creaz. Monaco Dance Forum, Salle de Garnier, 3 luglio 2oro. Dell'anno di redazione di questo studio, il 2017, è invece Para/Diso RevIsIted (2017), la riedizione del lavoro del 2010 per il Ballet de Marseille, di cui frattanto Greco e Scholten hanno rilevato la direzione. Cfr. Vigier 2oıo; Inbar-Volkov 20I7, e dossier online dello spettacolo: http://www.ickamsterdam.com/en/ productions/previous-productions/you-paradiso-I2. 
Purgatorio solistico, già prossimo ai climi del Paradiso; coscienti altresì di non potere restituire senza meno un'immagine celestiale del regno di dio, perchè partecipi di un pudore laico -comune a tutta la modernità - circa idee di beatitudine totale alla cui seduzione sembra aver ceduto, in danza, solo il settore più candido di certo neoclassicismo, i coreografi decidono di decostruire l'idea del Paradiso (e di avvicinarla alle distopie della realtà contemporanea) con mezzi che possono ricordare, curiosamente, la versione di inferno realizzata svariati anni prima da Paul Taylor in Dante Variations (che analizzerò nella seconda parte di questo studio): dispiegando, nei costumi, nelle scenografie e nel light design una poetica dello scintillio, del brillo, della luccicanza che, se da un lato riprende le iridescenze ipnotiche descritte da Dante nella parte più luminica del poema, sembra alludere dall'altra a un impensabile allineamento tra il paradigma del Paradiso cristiano e il glitter della mondanità recente, con le sue feste e mode e glamours: i "troppi paradisi" cui, in un romanzo del 2006, Walter Siti (Siti 2006) ha ascritto infallibilmente il deterioramento di tutte le qualità umane sfondo del totalitarismo spettacolare e del suo ricco catalogo di jouissances.

Il correlativo del procedimento paradigmatico che spinge Dante a fare di ogni beato la versione assoluta di una benemerenza morale o spirituale, è, in youPara/Diso, l'assegnazione a ogni interprete del compito di incarnare cineticamente sei dei molti attributi "superlativi" che si riuniscono nella costellazione isterica del pensiero positivo come garanzie di successo sociale, esistenziale o commerciale (cosmico, puro, divino, generoso, assoluto, futuristico) e di tradurli maniacalmente nella formula autogenica "I am cosmic, I am pure", ecc.). L'autopresentazione di queste allegorie sovreccitate sembra ricordare il tipo di deformazione antropologica, compiuta nel segno di una definitiva adesione autogenica del soggetto alla categoria del "prodotto di lusso", descritto da una parte della sociologia recente. ${ }^{68}$

A ulteriore conferma che questo trattamento allegorico punta di fatto a iscrivere lo scenario di appagamento metafisico, il dispositivo di gloria del Paradiso dantesco ${ }^{69}$ nell'adesione del soggetto postmoderno agli alti valori di effettismo del sistema del capitalismo autoritario, e ai molti effetti speciali che

68. Cfr. Verdú 2007. Negli ultimi anni si è dato in danza un certo rinnovamento dell' interesse per gli automatismi paradisiaci dell'universo dei consumi e per la metamorfosi dell'estasi mistica in statuto oggettuale (o oggettificato) del soggetto occidentale. Sintomaticamente si è data, nella prassi della danza post-concettuale, una certa profusione di glitter, oro, paillettes, lamé e altri espedienti materici volti a suggerire un certo tipo di "eccitazione luminica" del corpo danzante o del suo supporto spaziale: se ne ha sentore nel lavoro, tra gli altri, di Fagarazzi \& Zuffellato (Io Lusso, 2008), Alexandra Waierstall (Matter of Ages, 20I4), Christian Rizzo (Et pourquoi pas 'bodymakers', falbalas', bazaar', etc etc,?, 200I), Wim Vandekeybus nieuwZwart, 2009) Eulàlia Bergadà (Gold Dust Rush, 2016).

69. Su questo tema, soprattutto in merito alla carola dei beati nel Cielo del Sole ( $P d X-X I V)$, cfr. Catelli 2008; Ardissino 2009; Fratini 2014. 
lo corredano, tutto il materiale di movimento sembra oscillare tra esaltazione e prosternazione: rotazioni, elevazioni, sospensioni. L’ondeggiamento delle cortine iridescenti disegnate da Minsuk Cho non fa che amplificare questo parossismo cinetico di mobilitazione generale, di gioia incondizionale, di psicodelia spiccia. E che la letizia perfetta delle moltitudini inneggianti si riduca qui alla solitudine narcisista di un soggetto umano immiserito dalle sue ansie di perfezione, trova un'espressione perfetta nell'inno alla gioia beethoveniano eseguito nella versione più dimessa possibile, dalla voce off di un solista che lo canta a cappella stonandolo liberamente.

Umano troppo umano, e pertanto decisamente inumano, il Paradiso di Greco e Scholten non può che disabitarsi, nel finale, per fare posto al lungo fade in, fino all'incandescenza, di un circolo delle stesse lampadine che, in $\mathrm{Hell}$, avevano rappresentato la porta dell'Inferno: ${ }^{70}$ accecante bagliore elettrico, ancora sulle note dell'Inno alla Gioia, di un oggetto artificiale che è l'unico residuo degli splendori invocati da Dante nel finale di Paradiso XXXIII, e immagine terminale di un potere immoto e spettacolare preposto a tutto questo universo di agitazioni, di sovreccitazioni ridicole. Sulla scia di questa decostruzione del paradiso, Greco/Scholten non sembrano insensibili all'ipotesi che una lettura finale (o meglio, terminale) di Dante debba darsi nella chiave postindustriale e apertamente comica del montaggio di attrazioni.

La Commedia (20II), ${ }^{71}$ ipotesi di un'antologia di frammenti dalla trilogia, è anche la sintesi precoce di una propensione a leggere la materia dantesca secondo le logiche del Neo-circo e della Clownerie, che andrà popolarizzandosi precisamente nella prassi performativa dei primi anni 'ıo. Realizzato nella versione site-specific per eseguirsi sullo sfondo troneggiante dei Sette Palazzi Celesti (2004) le torri installate da Anselm Kiefer nell'Hangar Bicocca di Milano, La Commedia è in qualche modo l'esibizione slapstick, condotta da un maestro di cerimonie, di sette allegorie viventi (una per ognuno dei giorni della settimana) condannate a dare spettacolo delle false perfezioni (e dei veri vizi) in cui si avvita su se stessa la nostra relazione alienata con il tempo, e a coprirsi progressivamente dell'escrescenza, tra ridicola e peccaminosa, dei nasi rossi che ne denunciano la vocazione pagliaccesca. Lunedí sarà così un tipo atrabiliare, Martedí una diva egocentrica, Sabato un ballerino di club, ecc. L'allegoria della Domenica, di aspetto parodicamente cristologico, accompagnata da Let the Sunshine in del musical Hair (1979), sembra rimandare alle utopie del 1968 come a una promessa di Paradiso (fu l'anno, d'altronde, di Paradise Now del Living Theater) che preannunciava, senza volerlo, le distopie celestiali di quarant'anni dopo.

70. Durante lo spettacolo, lo stesso circolo di lampadine è già apparso debolmente, dietro i tendaggi della scenografia, a evocare le carole di luce nel Cielo del Sole di $P d X$.

7I. Prod. ICKamsterdam, creaz. Hangar Bicocca, Milano, 23 giugno 2011. 
Quella che nelle intenzioni di Kiefer è la rappresentazione architettonica dell'invincibile arroganza umana di concepirsi verticalmente e di costruire verso l'alto, ispirata dalla lettura del trattato misterico Sefer Hechalot (Libro dei palazzi) del $\mathrm{V}$ secolo (che descriveva il cammino ascendente di chi vuole approssimarsi all'essenza divina), addiviene nella poetica di Greco e Scholten a una metafora della compartimentazione dell'esperienza di vita in pacchetti di tempo, secondo la definizione di Franco "Bifo" Berardi (Berardi 2015), in segmenti intensivi di prestazione, consumo, godimento, esaltazione.

De La Commedia si realizzerà una versione ulteriore per spettacolo di strada, presentata in occasione dell'apertura dello Stedeljik Museum di Amsterdam, sullo spiazzo antistante il museo e con un allestimento di fortuna, in cui la cultura "bassa" del circo si staglia calcolatamente sulla trasparenza della facciata di vetro del museo come dispositivo di cultura "alta", e in cui la poetica di Greco e Scholten sembrerà insistere su una strategia velatamente trash di desublimazione o abiezione dei simboli che avevano contrassegnato la trilogia (la porta di Hell vi appare girata, l'albero montato su un lato alla bell'e meglio, le chitarre sono raggruppate in un angolo): la trilogia dantesca più pensosa, forse la più eloquente tra quelle realizzate nel contesto della danza contemporanea, non può terminare coerentemente il travaglio del metadiscorso cui ha affidato un'ipotesi generale di trascrizione danzata della Divina Commedia che con quest'ultimo atto di smantellamento o resa incondizionale di Dante alla postmodernità; di con-fusione circense e appiedata - spettacolarmente orizzontale - del poema e di tutti i suoi oltremondi. Almeno per quanto riguarda la danza, la Divina Commedia non tornerà mai più ai luoghi metafisici in cui si era creduto di trovarla.

Autoaccreditati per una lettura dell'escatologia dantesca che interpella le inquietudini escatologiche occulte a loro volta nel paradigma della danza contemporanea, Greco e Scholten sono anche, a tutti gli effetti, gli interpreti ultimi e probabilmente definitivi di una volontà di integrazione esegetica della Divina Commedia ai mezzi e formati tradizionali della danza d'arte. A partire da ora si darà, nella storia degli adattamenti danteschi, una specie di distrofia strutturale: molteplici linee di fuga e riassetti esegetici (quasi sempre nell'ordine di un drastico rilascio delle tensioni ermeneutiche) il cui effetto più evidente è stato l'alleanza mistica (e ampiamente mistificata) della lettera dantesca con tutte le prassi residuali, con tutti i formati terminali che la danza ha corteggiato nella postmodernità avanzata: metadiscorsivi, festivi, e a volte festosamente metadiscorsivi. Inflazione di un revival dantesco, quella degli ultimi anni, che come tutti i processi di banalizzazione segna una vittoria della quantità sulla qualità, perchè se evita di suo il rischio di trarre da Dante la benchè minima vertigine esegetica (e ha in qualche caso l'arroganza di in- 
scenarlo tale e quale nello splendore medievaleggiante del technicolor), appare d'altra parte dedita al tic culturale di invocarlo con ostinazione.

$\mathrm{Ma}$ è proprio questa insistenza, così essenziale all'inanità poetica propalata quasi senza eccezioni dal revival che ho detto, ciò che più energicamente fa la pertinenza paradossale della Weltansschaung dantesca alla definizione dell'epopea della danza contemporanea (la sua apoteosi o, secondo i punti di vista, la sua catastrofe), ben al di là di quanto il discorso di quella danza su di sé (e incidentalmente su Dante) voglia ammettere. È dantesca suo malgrado, la produzione degli ultimi anni - e dantesca malgrado la smidollata convinzione di poter realizzare in maniera congruente, di poter totalizzare il senso della Commedia - perchè in fondo travisa Dante con la stessa forza con cui travisa se stessa, e perchè il suo errore fondamentale è, a conti fatti, di contemplare come un paradiso l'inferno che dispiega (di interpretare per esempio come un approccio estetico definitivo il suo disorientamento estetico radicale, come una cartografia meditata l'intorno di arbitrio artistico in cui si muove); o meglio ancora, di non intendere che, se Dante può ormai giustificare ogni tipo di impromptu formale, è grazie a una stagnazione essenziale, a una deliquescenza delle poetiche (e ai "fermenti" totalmente funzionali che se ne producono) che non consente già più di distinguere l'alto e il basso: palude definitiva (direbbe Manganelli) in cui la maggior parte delle utopie e buone intenzioni di cui era lastricato il cammino della danza moderna, approdate a una realizzazione troppo completa, o troppo facile, o troppo letterale, si derealizzano per saturazione - senza speranza. Che persino dopo l'avventura poetica di Emio Greco (o chissà a causa di essa) la danza attuale non sappia neanche dire l'Oltremondo che, mentre pensa ancora di poterlo rappresentare, è la vera sostanza della sua escatologia. 


\section{BibliografiA}

Agamben, G., 2007, Il Regno e la Gloria. Per una genealogia teologica dell'economia e del governo. Homo Sacer, II, 4, Vicenza, Neri Pozza.

—, 20II, Altissima povertà. Regole monastiche e forma di vita. Homo Sacer, IV, I, Milano, Neri Pozza.

Allik, K., e Mulder, R., I99I, Electronic Purgatory. Eine digitale Musik-TheaterKomposition für Schauspieler, Toronto.

Ardissino, E., 2009, "Liturgia della gloria. La danza dei sapienti", Tempo Liturgico e tempo storico nella "Commedia" di Dante, Roma, Libreria Editrice Vaticana.

Auerbach, E., I966, "Figura” (1944), Studi su Dante, Milano, Feltrinelli, pp. 174-220.

Auslander, P., 1999, Liveness: Performance in a mediatized culture, London, Routledge.

Barolini, T., I992, The Undivine Comedy: Detheologizing Dante, Princeton, Princeton University Press.

Bartosik, K., 20II, "Technogenderbody”, Body, Space and Technology I, n. 2, online. $<$ people.brunel.ac.uk/bst/voloıo2/kimberlybartosik.html>

Baudrillard, J., I98I, Simulacres et simulations, Paris, Galilée.

Berardi, F. "Bifo", 2015, Heroes. Mass Murder and Suicide, New York, Verso Books.

Bertin, J., e Bertin, S., 20I4, Chevaux de souffrance. Les marathons de danse en Europe (I93I-1960), Le Mans, Éditions Cénomane.

Braida, A., e Calè, L. (a cura di), 2007, Dante on View: The Reception of Dante in the Visual and Performing Arts, Farnham, Ashgate Publishing.

Capaldi, D., 2006, "L'Inferno sul corpo", Dante. Rivista internazionale di studi su Dante Alighieri, III, pp. I89-I9I.

Carlson, C., 200I, Le Soi et le Rien, Paris, Actes Sud.

Catelli, N., 2008, "Coreografie paradisiache. Le danze dei sapienti (Par. X-XIV)", L'Alighieri. Rassegna dantesca, 49, n.s. XXXII, pp. II9-I38.

Cavallaro, A., 2002, Prefiguring Cyberculture: an Intellectual History, Cambridge (Mass.), MIT Press.

Delcourt, Thierry, 2015, Carolyn Carlson, de l'intime à l'universel, Arles, Actes Sud.

Depestel, D., e Vanderbeeken, R., 2015, Bastard or Playmate? Adapting theatre, mutating media and the contemporary performing arts, Amsterdam, Amsterdam University Press.

Didi-Huberman, G., 2002, L'image survivante. Histoire de l'art et temps des fantômes selon Aby Warburg, Paris, Les Editions de Minuit.

Dixon, S., 2007, Digital Performance. A History of new media in theater, dance, performance art, and installation, Cambridge (Mass.), MIT Press.

Donnarumma, M., 2012, "Performing Proprioception and Effort in 'Hypo Chrysos', an Action Art Piece for the XTH Sense", Econtact. Biotechnological Performance Practices, I4, n. 2, online. <http://econtact.ca/I4 2/donnarumma hypochrysos. html>

Dussel, E., 1996, Philosophy of Liberation, New York, Humanity Books.

Everson, J., 2007, "From Dante to Dante Sonata", in Braida-Calè 2007: 53-64.

Farber, P. M. (a cura di), 2015, The Wall in Our Heads: American artists and the Berlin Wall (Goethe-Institut Washington DC, October 25-December 25), catalogo, Washington.

Filiberti, I., 2006, "Les corps et ses lumières", Risorgimento for Hell, I, Amsterdam, Emio Greco / PC, pp.I2-I4. 
Forsythe, W., I991, "With a spider inside", La Parola alla danza: Cullberg, Linke, Forsythe, Wilson, incontri a cura di Marinella Guatterini, Milano, Ubulibri (Lezioni milanesi. Quaderni della Scuola d'Arte Drammatica Paolo Grassi, 3), pp. 49-62.

-, e Haffner, N., 2012, Improvisation Technologies. A Tool for the Analytical Dance Eye, New York, Art Pub Incorporated.

Franko, M., 2002, The Work of Dance. Labor, Movement and Identity in the I93os, Middletown, Wesleyan University Press.

Fratini, R., 20I4, "Vacabimus. Appunti per una cinesiologia dantesca", Danses imaginades, danses relatades. Paradigmes iconogràfics del ball des de l'antiguitat clàssica fins a l'edat mitjana, a cura di L. Buttà, J. Carruesco, F. Massip, E. Subías, Tarragona, Institut Català d'Arqueologia Clàssica, pp. 85-I08.

Garafola, L., 20II, "Astonish me!: Diaghilev, Massine and the experimentalist tradition", The Ballets Russes in Autralia and Beyond, a cura di M. Carroll, Kent Town, Wakefield Press, pp. 5I-76.

Goldsmith, K., 2015, Escritura no-creativa. Gestionando el lenguaje en la era digital, Buenos Aires, Caja Negra.

Gorni, G., 20II, Dante: Storia di un visionario, Bari, Laterza.

Goumarre, L., 2006, "Au milieu du chemin de notre vie", Risorgimento for Hell, I, Amsterdam, Emio Greco / PC, pp. 34-36.

Grau, O., 2003, Virtual Art. From Illusion to Immersion, Cambridge (Mass.), MIT Press.

Greco, E., 2008, "Propos recueilli par Antoine de Baecque", [purgatorio] Popopera, programma di sala, Lyon, Maison de la Danse.

Grotowski, J., I968, Towards a Poor Theatre, London, Methuen.

Grunberg, A., 2008, "Emotions without Owner", Risorgimento for Purgatorio, 2, Amsterdam, Emio Greco / PC, pp. I2-I5.

Gumts, H. P., 2008, "Spektakel Müssen sein und werden begeistert gefeiert", Gießener Allgemeine (31/08/2008), online. <http://www.giessener-allgemeine.de/regional/ stadtgiessen/art71,22796>

Hayles, K. N., 1999, How We Became Posthuman: Virtual bodies in cybernetics, literature and informatics, Chicago, University of Chicago Press.

Heinold, T., 2006, "Wenn der Alltag nervt: Pralinen helfen", Nordabayern (I6/OI/2006), online. <www.nordbayern.de/kultur/wenn-der-alltag-nervt-pralinenhelfen-I.7167I4>

Ihde, D., 2002, Bodies in Technology. Electronic Meditations, vol. 5, Minneapolis, University of Minnesota Press.

Inbar, A., e Volkov, H., 20I7, "Madness is the most blissful place", intervista agli interpreti di Para/Diso RevIsIted, Glamcult (o9/or/20I7), online.

<http://glamcult.com/paradiso-revisited>

Johnson, B. (a cura di), I891, Poems of Leigh Hunt with Prefaces of some of his Periodicals, ed. facs., Whitefish Montana, Kessinger Publishing, 2008.

Kozel, S., 2007, Closer. Performance, technology, phenomenology, Cambridge (Mass.), MIT Press.

Kurzweil, R., 2000, The Age of Spiritual Machines, London, Penguin Books.

La Salvia, A., 20II, "Dantes Göttliche Komödie in Musiktheater", Göttliche, menschliche und teuflische Komödien: europäische Welttheater-Entwürfe im I9. und 20. Jahrundert, a cura di A. La Salvia e A. Gier, Bamberg, University of Bamberg Press, pp. 253-28I. 
Laban, R. Von, 1994, La maîtrise du mouvement, Marseille, Actes Sud.

Lansing, R., 20IO, The Dante Encyclopedia, New York, Routledge.

Maletič, V., 1987, Body Space Expression, Berlin, Mouton de Gruyter.

McCaw, D. (a cura di), 20II, The Laban Sourcebook, New York, Routledge.

Mitchell, W., 2003, Me++: The Cyborg Self and the Networked City, Cambridge Massachussets, MIT Press.

Moretti, F., 1994, Opere mondo. Saggio sulla forma epica dal Faust a Cent'anni di solitudine, Torino, Einaudi.

Negroponte, N., 1995, Being Digital, New York, Knopf Doubleday, 2015.

Néri, C., 2006, "Enfer Intérieure?", Risorgimento for Hell, I, Amsterdam, Emio Greco / PC, pp. 22-24.

Pask, E. H., 1982, The Ballet in Australia. The second act: 1940-1980, Oxford, Oxford University Press.

Pouillade, F., 2009, Le Désoeuvrement chorégraphique. Étude sur la notion d'oeuvre en danse, Paris, J. Vrin.

Regli, F., I838, Il Pirata. Giornale di Letteratura, Belle Arti, Mestieri, Moda e Varietà, vol. 4, Milano, Nervetti.

Roglieri, M. A., 200I, Dante and Music. Musical adaptations of the Commedia from the XVIth century to the present, Farnham, Ashgate.

Schechner, R., 1977, Performance Theory, London, Routledge.

Scholten, P., 2008, "On Purgatorio / popopera", Risorgimento for Purgatorio, 2, Amsterdam, Emio Greco / PC, p. I2.

Sharir, Y., I997, "Virtually Dancing", ArtNet, online. <http://www.art.net/-dtz/sharirr.html>

Siti, W., 2006, Troppi paradisi, Torino, Einaudi.

Smith, C., 1937, "Third of New Ballets Wins Long Applause”, Chicago Daily Tribune (23/12/1937), p. I9.

Smith-Autard, J. M., 20I4, Dance Composition: a practical guide for creative success in dance making, New York, Routledge.

Steyerl, H., 20I4, Los condenados de la pantalla, Buenos Aires, Caja Negra.

Testa, A., 1990, "Viaggio con Dante danzando Liszt", La Repubblica (2I/o8/1990), online. <http://ricerca.repubblica.it/repubblica/archivio/repubblica/I99o/o8/2I/ viaggio-con-dante-danzando-liszt.html>

Tsao, A., 2013, "San Francisco Ballet: Criss-Cross, Francesca da Rimini, Symphony in Three Movements", DanceTabs (22/04/2013), online. <http://dancetabs.com/ 2013/o4/san-francisco-ballet-criss-cross-da-rimini-symphony-in-3-san-francisco $>$

Turnbull, A., I999, "Carolyn Carlson", in Bremser, M., Fifty Contemporary Choreographers, New York, Routledge, pp. 55-57.

Verdú, V., 2007, Yo y tú, objetos de lujo. El personismo: la primera revolución cultural del siglo XXI, Madrid, Debolsillo.

Vigier, I., 2008, "The essence of human motion", Risorgimento for Purgatorio, 2, Amsterdam, Emio Greco / PC, p. 85.

- (a cura di), 2oro, You PARA/DISO. A guide to a Dance Performance, Amsterdam, Emio Greco / PC ICK.

Walker, A., 20II, We Are the Ones We Have Been Waiting For: Inner light in a time of darkness, London, Weidenfeld \& Nicolson.

Walter, C. S., 20I4, Dance, Consumerism and Spirituality, New York, Palgrave MacMillan. 
Wesemann, A., 1997, "Mirror Games with the New Media: the story of dance has always been the story of technology", Ballett International Tanz Aktuell, 8, n. 9.

Wittschier, H. W., 2004, Dantes Divina Commedia. Einführung und Handbuch: Erzählte Transszendenz, Berlin, Peter Lang.

Zicari, I., 2013, "A writing that dictates the choreography: Dante sonata by Frederick Ashton", Studia Musicologica, 54, n. 4 (december), pp. 4I7-429.

\section{Cronologia delle opere}

I825 Francesca d'Arimino: azione tragica in cinque atti, cor. Giacomo Serafini, mus. Saverio Mercadante

I825 Francesca da Rimini: ballo eroico in cinque atti, cor. Giovanni Calzerani e Giulio Viganò, mus. Vincenzo Schira

I838 Francesca da Rimini: azione tragica in cinque atti, cor. Giacomo Serafini, mus. Saverio Mercadante

I856 Francesca da Rimini: opera ballo in quattro atti, cor. ignoto, mus. Cesare Favetti I857 Francesca da Rimini: azione tragico-mimica in cinque atti, cor. Antonio Coppini, mus. Ferdinando Gualtieri

I9Is Francesca da Rimini: balletto neoclassico, cor. Mikhail Fokine, mus. Piotr Ilič

Tchaikovsky. Creaz. Teatro Mariinskij, San Pietroburgo

I937 Francesca da Rimini: balletto neoclassico, cor. David Lichine, mus. Piotr Ilič

Tchaikovsky. Creaz. Royal Opera House, Covent Garden, London

I940 Dante Sonata (d'après une lecture de Dante): balletto neoclassico, cor. Frederick

Ashton, mus. Franz Liszt. Creaz. Vic Wells Ballet, Sadler's Wells Theatre, London

I955 Francesca da Rimini: balletto neoclassico, cor. Valrene Tweedie, mus. Franz Liszt.

Creaz. National Theatre Ballet, National Theatre, Melbourne

I955 Francesca da Rimini: balletto neoclassico, nuova versione, cor. David Lichine, mus. Piotr Ilič Tchaikovsky. Creaz. Borovansky Ballet, Empire Theatre, Sidney I976 La divine comédie: balletto neoclassico, cor. Vittorio Biagi; mus. Bernard

Parmegiani. Creaz. Ballet de Lyon, Opéra de Lyon

I990 Dante Symphony: danza contemporanea, cor. Micha Van Hoecke; mus. Franz

Liszt. Creaz. L'Ensemble di Micha van Hoecke, Rocca Brancaleone (Ravenna

Festival), Ravenna

I99I Dante in the Elevator. Lindy's Divine Comedy. Ein Stück für I7 Personen und einen

Paternoster: performance installazione, cor. Lindy Annis, mus. AA.VV. Creaz.

Gebäude des Senators für Bau- und Wohnwesen, Berlin

I99I The Divine Comedy: installazione coreografica, cor. Regina Miranda, mus.

AA.VV. Creaz. Museo de Arte Moderno, Rio de Janeiro

1989-1992 Electronic Purgatory (Elektronische Fegefeuer). Eine digitale Musik-Theater-

Komposition für Schauspieler: teatro interattivo e musica elettroacustica, dir. Kristi

Allik e Robert Mulder, mus. AA.VV. Creaz. Festival Synthèse, Bourges (vers. 1989, titolo provvisorio Pentaprism); ACREC, Montréal (vers. 1992)

1993 Commedia: danza contemporanea, cor. Carolyn Carlson, mus. Michel Portal e René Aubry. Creaz. Carolyn Carlson Company, Deutschen Schauspielhaus, Hamburg

I996 Synchronicity Space. The Dante Project: Inferno, dir. Bill Conte, cor. Kryota Willberg, mus. "Tolve". Creaz. Taohouse, New York, 55 Mercer Street 
1999 Zwischen Himmel und Hölle. Eine multimediale Inszenierung nach Dante Alighieri: teatro multimediale, dir. Wolfram Lensen, mus. Katharina Dustmann. Creaz. Forum Interart, Zweckel, Alte Maschinenhalle

2004 Dante Variations: danza moderna, cor. Paul Taylor, mus. György Ligeti. Creaz. Paul Taylor Company, Yerba Buena Center Theater, San Francisco

2005 Double Points: Hell: assolo danza contemporanea, cor. Emio Greco, mus. AA.VV.

Creaz. Cia EG | PC, le Sujet à Vif - Festival d'Avignon, Avignon

2006 Hell: danza contemporanea, cor. Emio Greco e Pieter Scholten, mus. AA.VV. Creaz. Cia EG|PC, Festival Montpellier Danse

2006 Nel foco che gli affina - Reinigende Feuer: danza contemporanea, cor. André Gringas, mus. Jurgen De Blönde. Creaz. Stadttheater, Nurnberg

2008 Die Göttliche Komödie. Ein Spektakel aus Schauspiel, Tanz und Musiktheater nach Dante Alighieri: teatro-danza, dir. Thomas Goritzki, cor. Tarek Hassam, mus. AA.VV. Creaz. Stadttheater, Gießen

2008 [purgatorio] Popopera: danza contemporanea, cor. Emio Greco e Pieter Scholten, mus. Michael Gordon. Creaz. ICK, EG|PC, Holland Festival, Amsterdam

2008 [purgatorio] In Visione: assolo danza contemporanea, cor. Emio Greco e Pieter Scholten, mus. Johann Sebastian Bach. Creaz. Prod. ICK, Holland Festival, Amsterdam

2009 Dante's Divine Comedy: video performance, dir. Rene Migliaccio, vid. India Evans, mus. Michaela Stehlikova. Creaz. Blackmoon Theatre Company, International Fringe Festival, New York

2008-20Io Reading Dante (I, II, III); Infernal Paradise: videoperformance, dir. Joan Jonas. Creaz. Performa, Sidney Biennale (vers. I); Galleria Yvon Lambert, New York (vers. III)

2010 YouPara/Diso: danza contemporanea, cor. Emio Greco e Pieter Scholten, mus. AA.VV. Creaz. EG|PC, Monaco Dance Forum, Salle de Garnier, Munich 20II La Commedia: danza contemporanea, cor. Emio Greco e Pieter Scholten, mus. AA.VV. Creaz. ICKamsterdam, Hangar Bicocca, Milano

20II Divina.com. From here to Eternity: danza contemporanea con videoinstallazione, cor. Keith Ferrone, video Adi Da Samraj, mus. Pankow e Stefano Maurizi. Creaz. Museo del Bargello, Firenze

2012 La Commedia (vers. esterna): danza contemporanea, cor. Emio Greco e Pieter Scholten, mus. AA.VV. Creaz. ICKamsterdam, Stedelijk Museum, Amsterdam 2012 Hypo Chrysos: performance e videoinstallazione, dir. Marco Donnarumma, video Studiotime. Creaz. Cynetart fest - Metabody Project, Dresden 2012 Francesca da Rimini: balletto neoclassico, cor. Yuri Possokhov, mus. Piotr Ilič Tchaikovsky. Creaz. San Francisco Ballet, San Francisco Opera

2014 Francesca da Rimini: videodanza, dir. Tarik Abdel-Gawad, cor. Yuri Possokhov, mus. Piotr Ilič Tchaikovsky

2014 La Divine Comédie: video-animazione, dir. Simon Coté-Lapointe. Prod. Simon Coté-Lapointe, Paris

2015 Die Göttliche Komödie: teatro multimediale, dir. Sebastian Baumgarten, video Stefan Bischoff, mus. Jens Massel. Creaz. Shauspielhaus, Köln

2015 Passione: danza contemporanea, cor. Emio Greco e Pieter Scholten, mus. Johann Sebastian Bach. Creaz. Ballet National de Marseille, Opéra de Marseille 2017 Para/Diso RevIsIted: danza contemporanea cor. Emio Greco e Pieter Scholten, mus. AA.VV. Creaz. Ballet de Marseille, Opéra de Marseille 
